\title{
NUMERICAL STUDY OF OSCILLATORY REGIMES IN THE KADOMTSEV-PETVIASHVILI EQUATION
}

\author{
CHRISTIAN KLEIN, CHRISTOF SPARBER, AND PETER MARKOWICH
}

\begin{abstract}
The aim of this paper is the accurate numerical study of the KP equation. In particular we are concerned with the small dispersion limit of this model, where no comprehensive analytical description exists so far. To this end we first study a similar highly oscillatory regime for asymptotically small solutions, which can be described via the Davey-Stewartson system. In a second step we investigate numerically the small dispersion limit of the KP model in the case of large amplitudes. Similarities and differences to the much better studied Korteweg-de Vries situation are discussed as well as the dependence of the limit on the additional transverse coordinate.
\end{abstract}

version: September 27, 2018

\section{INTRODUCTION}

This work is concerned with the $2+1$ dimensional Kadomtsev-Petviashvili equation $(\mathrm{KP})$, given by

$$
\partial_{x}\left(\partial_{t} u+u \partial_{x} u+\epsilon^{2} \partial_{x x x} u\right)+\lambda \partial_{y y} u=0, \quad \lambda= \pm 1,
$$

where $(t, x, y) \in \mathbb{R}_{t} \times \mathbb{R}_{x} \times \mathbb{R}_{y}$ and where $\epsilon>0$ is a (small) scaling parameter, as introduced below. The case $\lambda=-1$ corresponds to the so-called KP-I model, whereas $\lambda=+1$ is usually referred to as KP-II equation. Both cases can be derived as models for nonlinear dispersive waves on the surface of fluids 24] (see also 22]). Thereby it is assumed that the propagation of the waves is essentially one-dimensional with weak transverse effects. Roughly speaking, KP-I describes the case when surface tension is strong, whereas KP-II is a good model for weak surface tension. Moreover, the KP type equations also arise as a model for sound waves in ferromagnetic media [42] and in the description of two-dimensional nonlinear matter-wave pulses in Bose-Einstein condensates, see e.g. [19] 23].

Notice that the KP equation has originally been derived in the following form

$$
\begin{aligned}
\partial_{t} u+u \partial_{x} u+\epsilon^{2} \partial_{x x x} u+\lambda \partial_{y} v & =0,\left.\quad u\right|_{t=0}=u_{\mathrm{I}}(x, y), \\
\partial_{y} u & =\partial_{x} v,
\end{aligned}
$$

where the second equation can now be seen as a constraint for the Cauchy problem given in the first line. Therefore we shall also impose for equation (1.1) initial data in the Schwartz class of smooth and rapidly decreasing functions, i.e.

$$
\left.u\right|_{t=0}=u_{\mathrm{I}} \in \mathcal{S}\left(\mathbb{R}_{x} \times \mathbb{R}_{y}\right),
$$

2000 Mathematics Subject Classification. 37K10, 35Q53, 34E05, 35Q55.

Key words and phrases. Kadomtsev-Petviashvili equation, nonlinear dispersive models, multiple scales expansion, modulation theory, Davey-Stewartson system.

We thank B. Dubrovin, E. Ferapontov, J. Frauendiener, and T. Grava for helpful discussions and hints. This work has been supported by the Wittgenstein Award 2000 of the second author. C.S. has been supported by the APART research grant funded by the Austrian Academy of Sciences. 
even though an interpretation of (1.1) in terms of a Cauchy problem seems not completely obvious at first sight, due to the appearing mixed derivative $\partial_{x t} u$. Nevertheless this is the usual approach when dealing with (1.1) and it is strengthened by the fact that in large parts of the literature one considers the KP model in its so-called evolutionary form

$$
\partial_{t} u+u \partial_{x} u+\epsilon^{2} \partial_{x x x} u+\lambda \partial_{x}^{-1} \partial_{y y} u=0,\left.\quad u\right|_{t=0}=u_{\mathrm{I}}(x, y) .
$$

Here, the anti-derivative $\partial_{x}^{-1}$ can be uniquely defined via

$$
\partial_{x}^{-1} f(x):=\frac{1}{2}\left(\int_{-\infty}^{x} f(\zeta) \mathrm{d} \zeta-\int_{x}^{+\infty} f(\zeta) \mathrm{d} \zeta\right),
$$

at least if $f(x)$ decays sufficiently fast to zero, as $x \rightarrow \pm \infty$, respectively. Alternatively $\partial_{x}^{-1}$ can be seen as a Fourier multiplier with the singular symbol $-\mathrm{i} / k_{x}$, as we shall do in the following.

In this paper we are interested in the accurate numerical simulation of the KP equation using a spectral scheme with preconditioning. To this end we remark that alternative numerical schemes for $2+1$ dimensional wave equations have been proposed in 11, 12. Moreover, earlier numerical studies of the KP equation can also be found in, e.g., 20, 25, 34, 44, 45, aiming mostly at the description of (interacting) solitons. In our work though the focus will be mainly on asymptotic regimes of the KP model. In particular we are concerned with the so-called dispersionless limit, i.e. the limit of (1.1) as $\epsilon \rightarrow 0$.

To motivate this study consider the unscaled (dimensionless) KP model

$$
\partial_{X}\left(\partial_{T} u+u \partial_{X} u+\partial_{X X X} u\right)+\lambda \partial_{Y Y} u=0,\left.\quad u\right|_{T=0}=u_{\mathrm{I}}(X, Y),
$$

where $(T, X, Y)$ are now considered as the natural scales of observation, or microscopic scales. In (1.6) we shall now introduce slowly varying solutions of the form, i.e.

$$
u=u(\epsilon T, \epsilon X, \epsilon Y), \quad 0<\epsilon \ll 1,
$$

where $\epsilon$ is a small scaling parameter, i.e. the microscopic/macroscopic scales ratio. These slow variations, for example, can be introduced via a class of corresponding initial data. Plugging (1.7) into (1.6) and denoting $(t, x, y)=(\epsilon T, \epsilon X, \epsilon Y)$, we obtain the scaled equation (1.1) with $\epsilon \ll 1$. In the following we shall always consider the KP equation in the form (1.1). The notion of the coordinates thus refers to the macroscopic sales.

In the formal limit $\epsilon \rightarrow 0$ we get the dispersionless KP equation (dKP), i.e.

$$
\partial_{x}\left(\partial_{t} u+u \partial_{x} u\right)+\lambda \partial_{y y} u=0 .
$$

Clearly this is a singular limiting procedure, which requires particular care. The analogous problem for the scaled Korteweg-de Vries equation (KdV), see equation (2.1) below, has been intensively studied by several authors, cf. [15, 29, 30, 31. More recently this problem has also been treated numerically in 16. In the KdV case, it is known that the solution of the Cauchy problem for smooth hump-like initial data is characterized by the appearance of a zone of rapid modulated oscillations [16. 39. A rigorous asymptotic treatment of these oscillations has been established, relying heavily on inverse scattering techniques and complete integrability. On the other hand, a complete mathematical description of the small dispersion limit for the KP model has not yet been achieved [5, 28. The purpose of this paper is thus to explore this problem numerically. Similar to KdV, it is expected that the dKP model will develop shocks in finite time. In this case, the formal limit (1.6) is 
no longer an accurate description of (1.1) as $\epsilon \rightarrow 0$. For small, but still non-zero $\epsilon$, the dispersive term $\propto \partial_{x x x} u$ in (1.4) presumably will smooth out these shocks by forming an oscillatory zone. This work provides numerically evidence for these facts.

More precisely the outline of this paper is as follows: In the next section we shall first recall some well known features of the KP equation. Also, we shall derive the Green's function of the corresponding linear model, which already gives some insight on the oscillatory nature of the considered equation. In Section 3 the numerical algorithm is presented and we shall test its performance for two cases of soliton propagation. In Section 4 we discuss the the asymptotic behavior for $\epsilon \ll 1$ in the particular case of small solutions, i.e. with amplitudes of order $\mathcal{O}(\epsilon)$. This yields an asymptotic description via the Davey-Stewartson system, which is studied numerically for the case $\lambda=1$. The corresponding asymptotic error to the KP-II equation, defined in the $L^{2}\left(\mathbb{R}^{2}\right)$ sense, is, within numerical precision, found to be $\mathcal{O}\left(\epsilon^{5 / 2}\right)$ which is remarkable since it is exactly as expected from the corresponding rigorous $\mathrm{KdV}$ studies in 35. Finally we turn to the problem of small dispersion for solutions of order $\mathcal{O}(1)$ in Section 5 We shall numerically investigate the entropy solution of the dKP equation and the corresponding oscillatory behavior in the KP model, in particular its dependence on the $y$-coordinate. We also find numerically that the asymptotic $L^{2}\left(\mathbb{R}^{2}\right)$-error in this case is $\mathcal{O}\left(\epsilon^{3 / 2}\right)$, at least before the appearance of the first shock in the corresponding dKP solution.

\section{Properties of the KP EQuation}

2.1. Preliminaries. In the context of water waves, the KP equation can be seen as a two-dimensional generalization of the celebrated KdV equation 22, i.e.

$$
\partial_{t} u+u \partial_{x} u+\epsilon^{2} \partial_{x x x} u=0,\left.\quad u\right|_{t=0}=u_{\mathrm{I}}(x) .
$$

Clearly, any solution to KdV is a $y$-independent solution to the KP equation, forming the KdV sector of (1.1). Indeed similar to $\mathrm{KdV}$, the $\mathrm{KP}$ model belongs to the class of integrable systems and is known to conserve an infinite number of quantities, among them mass

$$
\mathrm{M}[u(t)]:=\int_{\mathbb{R}^{2}} u^{2}(t, x, y) \mathrm{d} x \mathrm{~d} y
$$

and energy

$$
\left.\mathrm{E}[u(t)]:=\frac{1}{2} \int_{\mathbb{R}^{2}}\left(\partial_{x} u(t, x, y)\right)^{2}-\lambda\left(\partial_{x}^{-1} \partial_{y} u(t, x, y)\right)^{2}-\frac{\epsilon^{2}}{3} u^{3}(t, x, y)\right) \mathrm{d} x \mathrm{~d} y,
$$

as well as momentum etc., cf. [1 for more details. Again similar to KdV, explicit solutions to the KP model can be obtained via inverse scattering methods. In the following though we shall not go into more details on this important topic, arising in the study of integrable systems, and rather refer to, e.g., 3, 33] and the references given therein.

Despite the apparent similarity of KP-I and KP-II, the two equations behave rather differently both qualitatively and quantitatively. For example, only the KP-I has the advantage of having an energy which is positive definite in the leading order terms. Additionally, unlike the KP-I model, the KP-II equation does not admit so-called lump solitons. Generally speaking, a KP soliton is a solution of the form

$$
u(t, x, y)=u\left(x-t v_{x}, y-t v_{y}\right)
$$


with (constant) group velocity $v_{\mathrm{g}}=\left(v_{x}, v_{y}\right) \in \mathbb{R}^{2}$. Clearly, any $\mathrm{KdV}$ soliton is also a KP soliton solution. We also remark that both KP models bear several differences in what concerns the stability or instability of solitons, $c f$. 3, 21] and the references given therein. The single lump soliton to (1.1), with $\lambda=-1$, has the following form (here, we set $\epsilon=1$ for simplicity)

$$
u(t, x, y)=\frac{24 c\left(1-c(x-3 c t)^{2}+3 c^{2} y^{2}\right)}{\left(1+c(x-3 c t)^{2}+3 c^{2} y^{2}\right)^{2}}, \quad c \in \mathbb{R} .
$$

Note that the lump soliton only decays algebraically in both spatial directions, as $|x|,|y| \rightarrow \infty$. On the other hand both equations admit so-called line soliton solutions. These are solitons which are infinitely extended in one spatial direction and which exponentially decay in the other direction. The simplest solution of this kind is a $y$-independent 1 -soliton of the $\mathrm{KdV}$ equation (again $\epsilon=1$ for simplicity)

$$
u(t, x)=\frac{12}{\cosh ^{2}\left(x-x_{0}-4 t\right)}, \quad \text { with } x_{0} \in \mathbb{R} \text { fix. }
$$

Solutions which are even better localized in $x$ and $y$ cannot be expected (see also 7). Indeed it is a common feature of both KP-I and KP-II that, even if the initial data is a Schwartz function the corresponding solutions $u(t)$ for $t>0$ in general will not stay in the Schwartz class, unless $u_{\text {I }}$ satisfies in addition an infinite number of constraints. Rather it is known that, for generic initial data, the solutions $u(t)$ will develop "tails" decaying only algebraically in certain spatial directions. The reason for this behavior is already seen on the level of the Green's function for the linear KP equation, as we shall discuss in the next subsection.

2.2. The linear KP equation. In the following we shall study the linear part of the KP equation, i.e.

$$
\partial_{x}\left(\partial_{t} u+\epsilon^{2} \partial_{x x x} u\right)+\lambda \partial_{y y} u=0,\left.\quad u\right|_{t=0}=u_{\mathrm{I}}(x, y) .
$$

for smooth (finite mass) solutions $u(t) \in L^{2}\left(\mathbb{R}^{2}\right) \cap L^{1}\left(\mathbb{R}^{2}\right)$. If we denote by

$$
(\mathcal{F} u(t))\left(k_{x}, k_{y}\right) \equiv \widehat{u}\left(t, k_{x}, k_{y}\right):=\int_{\mathbb{R}^{2}} u(t, x, y) \mathrm{e}^{-\mathrm{i}\left(k_{x} x+k_{y} y\right)} \mathrm{d} x \mathrm{~d} y,
$$

the Fourier transform of $u(t)$ w.r.t $x$ and $y$, the above given equation (2.7) is equivalent to

$$
\mathrm{i} k_{x} \partial_{t} \widehat{u}+\epsilon^{2} k_{x}^{4} \widehat{u}-\lambda k_{y}^{2} \widehat{u}=0 .
$$

In order to pass to the evolution form (1.4) we need to be able to apply the singular multiplier $-\mathrm{i} / k_{x}$ to this equation, to obtain

$$
\partial_{t} \widehat{u}+\mathrm{i}\left(\frac{\lambda k_{y}^{2}}{k_{x}}-\epsilon^{2} k_{x}^{3}\right) \widehat{u}=0 .
$$

Having in mind that $\widehat{u}(t) \in C_{\mathrm{b}}\left(\mathbb{R}^{2}\right)$, for $u(t) \in L^{1}\left(\mathbb{R}^{2}\right)$, this certainly requires that $\widehat{u}\left(t, 0, k_{y}\right)=0$, for all $k_{y} \in \mathbb{R}$. To ensure this, we shall impose the following condition on the initial data

$$
\int_{\mathbb{R}} u_{\mathrm{I}}(x, y) \mathrm{d} x=0,
$$

which, in Fourier space, is equivalent to $u_{\mathrm{I}}\left(0, k_{y}\right)=0$, for all $k_{y} \in \mathbb{R}$. In this case the (weak) solution of (2.9) is obviously given by

$$
\widehat{u}\left(t, k_{x}, k_{y}\right)=\mathrm{e}^{-\mathrm{i} t\left(\lambda k_{y}^{2} / k_{x}-\epsilon^{2} k_{x}^{3}\right)} u_{\mathrm{I}}\left(k_{x}, k_{y}\right),
$$


which satisfies $\widehat{u}\left(t, 0, k_{y}\right)=0$, for all $t \in \mathbb{R}$. The constraint (2.10) thus seems to be quite natural when passing to the formulation (1.4), cf. 6, 43. Moreover it can also be seen from the numerical example given in Section 3 that initial data which do not satisfy (2.10) become non-differentiable for any $t \neq 0$.

Remark 2.1. Alternatively one might also remark that (formally) integrating (1.1) w.r.t. to $x \in \mathbb{R}$ gives

$$
\int_{\mathbb{R}} \partial_{y y} u(t, x, y) \mathrm{d} x=0, \quad \forall y \in \mathbb{R}, t \neq 0,
$$

or, equivalently, $-k_{y}^{2} \widehat{u}\left(t, 0, k_{y}\right)=0$, a condition certainly fulfilled by (2.11), 2.10).

Of course $\widehat{u}\left(t, 0, k_{y}\right)=0$ is not sufficient to obtain a strong KP-solution (being differentiable in-time) which satisfies (2.9) point wise in Fourier space. To this end one would require a sufficient fast decay of $\widehat{u}\left(t, k_{x}, k_{y}\right)$, as $k_{x} \rightarrow 0$. Therefore most of the literature is concerned with the mild formulation (2.11), which is consequently translated to the non-linear model, via Duhamel's formula

$$
u(t, x, y)=U(t) u_{\mathrm{I}}(x, y)-\int_{0}^{t} U(t-s) u(s, x, y) \partial_{x} u(s, x, y) \mathrm{d} s .
$$

Here, $U(t)$ denotes the unitary group in $L^{2}\left(\mathbb{R}^{2}\right)$ defined via its symbol in Fourier space, i.e.

$$
\widehat{U}(t):=\exp \left(-\mathrm{i} t\left(\lambda k_{y}^{2} / k_{x}-\epsilon^{2} k_{x}^{3}\right)\right) .
$$

Following this approach, well posedness issues are usually studied in non-isotropic Sobolev spaces $H^{s_{1}}\left(\mathbb{R}_{x}\right) \times H^{s_{2}}\left(\mathbb{R}_{y}\right)$. It should be noted that local and global well posedness results are much more complete in the KP-II case than in the case of KP-I. The first result in this direction has been obtained in 8] proving, that the KP-II model is globally well posed for $u_{\mathrm{I}} \in L^{2}\left(\mathbb{R}^{2}\right)$, or $u_{\mathrm{I}} \in L^{2}\left(\mathbb{T}^{2}\right)$. As far as Sobolev regularity is concerned we shall only remark that the required index pair for KP-II is: $s_{1}>-1 / 3, s_{2} \geq 0$ [37, referring to [10, 32, 38, 43] for more details on these issues.

Remark 2.2. Note that the formal identification $\partial_{t x} u=\partial_{x t} u$ requires particular care if one does not consider solutions, which are differentiable in time 32. In this context we also remark that sometimes even weaker notions of solutions which are only defined to be distributional in-time, are taken into account, $c f$. [6].

In order to get more insight on the dynamics of the linear equation we first note that (2.11) implies

$$
u(t, x, y)=\frac{1}{4 \pi^{2}}\left(\mathcal{F}^{-1} \widehat{U}(t)\right) * u_{\mathrm{I}}(x, y),
$$

where $*$ denotes the convolution w.r.t. $x \in \mathbb{R}$ and $y \in \mathbb{R}$. In order to calculate the inverse Fourier transform of $\widehat{U}(t)$ we shall split the transformation in two parts via

$$
\mathcal{F}^{-1}\left(\mathrm{e}^{-\mathrm{i} t\left(\lambda k_{y}^{2} / k_{x}-\epsilon^{2} k_{x}^{3}\right)}\right)=\mathcal{F}^{-1}\left(\mathrm{e}^{-\mathrm{i} t \lambda k_{y}^{2} / k_{x}}\right) * \mathcal{F}^{-1}\left(\mathrm{e}^{\mathrm{i} t \epsilon^{2} k_{x}^{3}}\right) .
$$

In the following let us restrict to the case of solutions for times $t>0$, for simplicity. Next, consider the last factor on the r.h.s. of (2.16) and recall the Fourier representation of the so-called Airy function, i.e.

$$
\operatorname{Ai}(x)=\frac{1}{2 \pi} \int_{\mathbb{R}} \mathrm{e}^{\mathrm{i}\left(x k+k^{3} / 3\right)} \mathrm{d} k .
$$


It is well known, see, e.g., [2], that the function $\operatorname{Ai}(x)$ admits a series expansion in the form

$$
\operatorname{Ai}(x)=\frac{1}{3^{2 / 3} \pi} \sum_{n=0}^{\infty} \frac{\Gamma\left(\frac{n+1}{3}\right)}{n !}\left(3^{1 / 3} x\right)^{n} \sin \left(\frac{2(n+1) \pi}{3}\right) .
$$

Moreover $\operatorname{Ai}(x)$ obeys two completely different asymptotic limits as $x \rightarrow \pm \infty$, respectively, namely

$$
\operatorname{Ai}(x) \stackrel{x \rightarrow+\infty}{\sim} \frac{1}{2 \pi^{1 / 2} x^{1 / 4}} \mathrm{e}^{-\frac{x^{3 / 2}}{3}}, \quad \operatorname{Ai}(x) \stackrel{x \rightarrow-\infty}{\sim} \frac{1}{\pi^{1 / 2} x^{1 / 4}} \cos \left(\frac{2 x^{3 / 2}}{3}-\frac{\pi}{4}\right) .
$$

We consequently obtain, by substituting $k=k_{x}\left(3 t \epsilon^{2}\right)^{1 / 3}$, that

$$
\mathcal{F}^{-1}\left(\mathrm{e}^{\mathrm{i} t \epsilon^{2} k_{x}^{3}}\right)=\frac{1}{\left(3 t \epsilon^{2}\right)^{1 / 3}} \operatorname{Ai}\left(\frac{x}{\left(3 t \epsilon^{2}\right)^{1 / 3}}\right) \otimes \delta(y), \quad \forall t>0,
$$

in $\mathcal{S}^{\prime}\left(\mathbb{R}_{x} \times \mathbb{R}_{y}\right)$, i.e. the sense of tempered distributions.

On the other hand, computing the inverse Fourier transform of the first factor on the r.h.s. of (2.16) yields, after some lengthy computations, that (for $t>0$ )

$$
\mathcal{F}^{-1}\left(\mathrm{e}^{-\mathrm{i} t \lambda k_{y}^{2} / k_{x}}\right)= \begin{cases}\frac{4 t^{1 / 2}}{\left(4 x t+\lambda y^{2}\right)^{3 / 2}}, & \text { if: } 4 x t+\lambda y^{2}>0 \\ 0 & \text { else. }\end{cases}
$$

Again this has to be interpreted as a distribution on Schwartz space. (We note that formula (2.21) is equivalent to the one found in 6], where only the KP-I case is considered though.) Expression (2.21) yields the Green's function for the linear partial differential operator

$$
L f:=\left(\partial_{x t}+\lambda \partial_{y y}\right) f=0, \quad \lambda= \pm 1 .
$$

Note that $L f=0$ is a hyperbolic PDE as can be seen by introducing the coordinates $\alpha=x+t$ and $\beta=x-t$, and transforming (2.22) into

$$
\left(\partial_{\alpha \alpha}-\partial_{\beta \beta}+\lambda \partial_{y y}\right) f=0, \quad \lambda= \pm 1 .
$$

This is nothing but the standard $2+1$ dimensional wave equation, where $\alpha$ or $\beta$ take on the role of a "time variable" for $\lambda=-1$ and $\lambda=1$, respectively. Since the operator $L$ can also be interpreted as the linear part of the dKP equation (1.8), imposing at time $t=0$ an initial condition of the form (1.3) indeed furnishes a characteristic Cauchy problem. Moreover, the space-time region determined by $4 x t+\lambda y^{2}=0$ is also a characteristic which explains the divergences of the Green's function (2.21) there.

Combining (2.15) with the formulas (2.20) and (2.21) we can draw several important consequences:

- The $y$-dependence of the solutions to the linear KP equation (2.7) is completely non-oscillatory and has a rather slow algebraic decay as $|y| \rightarrow \infty$.

- For any time $t>0$ we obtain infinitely extended tails of the solution within the region determined by $4 x t+\lambda y^{2}>0$, for $t>0$.

- The oscillatory behavior of the linear KP equation is governed by the Airy function. In our scaling this yields oscillations with wave length $\mathcal{O}(\epsilon)$ in the $x$-direction, as can be seen from (2.19). 


\section{The NUMERICAL ALGORITHM}

3.1. A Spectral approach with preconditioning. We are interested in the numerical solution of the KP equation for rapidly decreasing, smooth initial data $u_{\mathrm{I}}$. To study the zone of fast oscillations, due to $\epsilon \ll 1$, it is convenient to use a periodic setting of sufficiently large period. This allows for the use of spectral methods which are of high accuracy and efficiency because of their excellent approximation properties for smooth functions and the existence of fast algorithms for the Fourier transformation. The power law tails of the solution, as encountered in the preceding section, however will lead to an increasing number of "echoes" as time goes on, due to the chosen periodic domain of computation. For sufficiently large periods though these echoes will be small on the studied time-scales and they will not influence the analysis of the KP oscillations.

We use here an adapted version of Trefethen's code for the KdV equation (Chap. 10 in [40]) which is available at 41. The basic idea of the code is the use of a discrete Fourier transform in the spatial coordinates as well as of an integrating factor such that the time derivative is the only linear term appearing in the equation. This preprocessing, as introduced in [18, leads to an equation without a stiff part and allows for larger time steps. The method is slightly more efficient than the semi-implicit approach of [12, or time splitting techniques such as 17] e.g.. More importantly, however, it allows for the use of higher order time discretizations which are convenient in the context of strong gradients, as needed in Subsection 5.1 below. Keep in mind that an $n$-th order approach introduces a numerical dispersion of the order $(\Delta t)^{n+1}$, which leads to a "pollution" of the high spatial frequencies in the numerical time evolution. On the other hand though we need these high frequencies to resolve numerically the solution near a gradient catastrophe in the solution of the dKP model. Thus it is helpful to be able to apply a higher order method in the time discretization within an efficient approach which allows to deal with $2+1$ dimensional settings.

As before, let $\widehat{u}(t)$ be the Fourier transform of $u(t)$ and denote by $\widehat{u^{2}}$ the Fourier transform of $u^{2}$. Then the Fourier transformed KP equation (1.1) reads

$$
\partial_{t} \widehat{u}+\left(\frac{\mathrm{i} \lambda k_{y}^{2}}{k_{x}+\mathrm{i} \lambda 0}-\epsilon^{2} \mathrm{i} k_{x}^{3}\right) \widehat{u}+\frac{\mathrm{i}}{2} k_{x} \widehat{u^{2}}=0 .
$$

Equation (3.1) has to be regularized for $k_{x}=0$ in order to give numerically sense to the term $-\mathrm{i} / k_{x}$. Obviously division by zero is not allowed, even if the result would make sense analytically in a certain limiting procedure. To carry out such a limit numerically one has to use appropriately chosen finite numbers. Here this is done by adding to $k_{x}$ in the denominator a small imaginary part of appropriate sign (corresponding to $\lambda$ ). In the numerics we add the smallest floating point number which Matlab can represent, $2.2 \ldots 10^{-16}$. Equation (3.1) is then equivalent to

$$
\partial_{t}\left(\mathrm{e}^{\mathrm{i} t\left(\lambda k_{y}^{2} /\left(k_{x}+\mathrm{i} \lambda 0\right)-\epsilon^{2} k_{x}^{3}\right)} \widehat{u}\right)+\frac{\mathrm{i} k_{x}}{2} \mathrm{e}^{\mathrm{i} t\left(\lambda k_{y}^{2} /\left(k_{x}+\mathrm{i} \lambda 0\right)-\epsilon^{2} k_{x}^{3}\right)} \widehat{u^{2}}=0 .
$$

To solve equation (3.2) numerically we use the Fast Fourier Transform (FFT) in MATLAB for the dependence on the spatial coordinates and a fourth-order RungeKutta method for the time integration for the reasons given above. The important term in this integration is $\mathrm{e}^{-\mathrm{i} \Delta t\left(\lambda k_{y}^{2} /\left(k_{x}+\mathrm{i} \lambda 0\right)-\epsilon^{2} k_{x}^{3}\right)}$ as can be seen, e.g., from the simple time discretization

$$
\widehat{u}(t+\Delta t)=\mathrm{e}^{-\mathrm{i} \Delta t\left(\lambda k_{y}^{2} /\left(k_{x}+\mathrm{i} \lambda 0\right)-\epsilon^{2} k_{x}^{3}\right)}\left(\widehat{u}(t)-\frac{\mathrm{i} \Delta t k_{x}}{2} \widehat{u^{2}}\right) .
$$


Since we use an explicit method for time integration, stability is an issue. We find that due to the integrating factor, time steps of the order $\Delta t \sim 1 /\left(N_{x} N_{y}\right)$ lead to a stable time evolution. In the following, the empirically found values for the time steps needed in the respective computation are always given.

As already discussed in Section 2 we require our initial data to be subject to the constraint (2.10). In the periodic setting we analogously impose

$$
\int_{-\pi L_{x}}^{\pi L_{x}} u_{\mathrm{I}}(x, y) \mathrm{d} x=0
$$

where $2 \pi L_{x}$ is the period in $x$. For such data we solve numerically for the function $\hat{v}$ defined by $\hat{u}=i k_{x} \hat{v}$ to reduce numerical errors.

Remark 3.1. The numerical code is also able to propagate initial data which do not satisfy the constraint (3.4), which however yields a non-smooth solution in arbitrary short times. The reason for this is the analytical behavior of the term $\mathrm{e}^{-\mathrm{i} t \lambda k_{y}^{2} /\left(k_{x}+\mathrm{i} \lambda 0\right)}$ appearing in (3.3). Indeed, for $k_{x}=0$, this term is numerically equal to 0 , unless $k_{y}=0$ where it is equal to 1 . This leads to a continuous but not differentiable solution at $x=0$, see Fig. [1

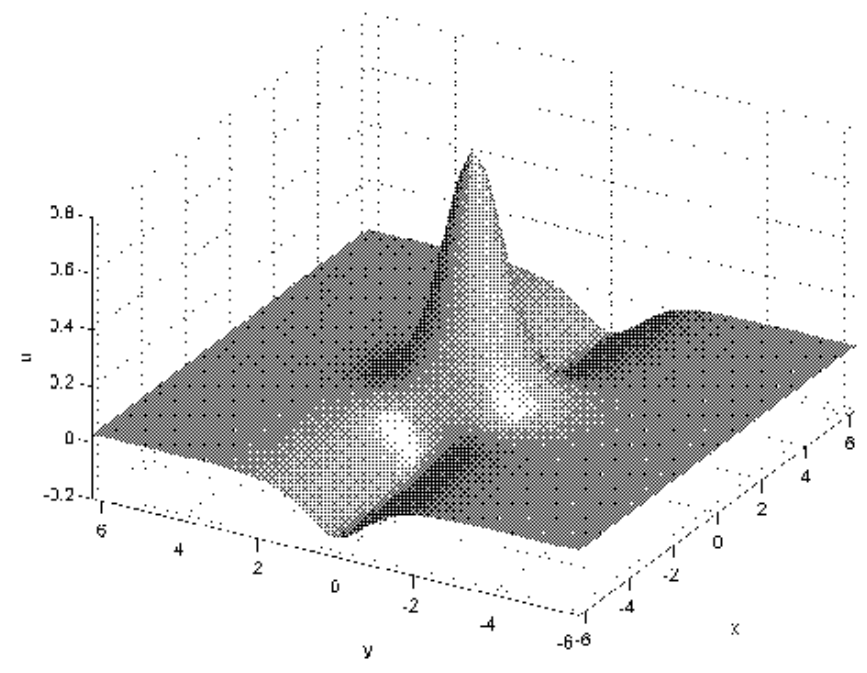

FiguRE 1. Solution at time $t=4.8 \times 10^{-4}$ to the KP-I solution with initial data $1 / \cosh ^{2}\left(\sqrt{x^{2}+y^{2}}\right)$ which are not subject to the constraint (3.4).

3.2. Numerical test cases on solitons. To test the accuracy of the code we compare, for the different time steps, the numerical solution to an explicitly known exact solution of the KP equation. Since the KP equation is completely integrable, large classes of explicit solutions are known. The most discussed cases certainly are the above-mentioned solitons. We use here the 1-soliton (2.6). The propagation of this solution formally does not test the $y$-dependent terms in the KP equation. However, this is only partially true since the line solitons are known to be unstable against perturbations in the case of the KP-I equation. Since unavoidable numerical errors provide some form of perturbation, the propagation of (2.6) also tests the 
$y$-dependent terms in the numerical implementation, see the example below. The reason for the use of a $y$-independent solution is that line solitons with an explicit $y$-dependence, $c f$. 33. will not be periodic in $y$.

The test of the propagation of the 1-soliton initial data is performed for the KPI model in the following setting: The $x$-coordinate takes values in the interval $\left[-\pi L_{x}, \pi L_{x}\right]$, where the length $L_{x}$ is always chosen such that the coefficients for the initial data are of the same order as the rounding error for high frequencies ${ }^{1}$. This reduces the error due to the non-periodicity of the initial data at the interval junctions to the order of the rounding error. The choice of the corresponding length $L_{y}$ is not important in this context, thus we shall choose it to be of the same value as $L_{x}$. We use the 1 -soliton solution at time $t=0$ with $L_{x}=10$ and $x_{0}=-L_{x}$ in the initial data and determine for each time step the difference between the exact and the numerical solution. The computation is carried out with $N_{x}=2^{11}$ and $N_{y}=2^{7}$ modes (we will always use powers of 2 here since the FFT algorithm is most efficient in this case, but this is not necessary) and $4 \times 10^{3}$ time steps for $t \in[0,6]$. The $L^{\infty}$ norm of the difference between the numerical and the exact solution is shown as a function of time in Fig. 2

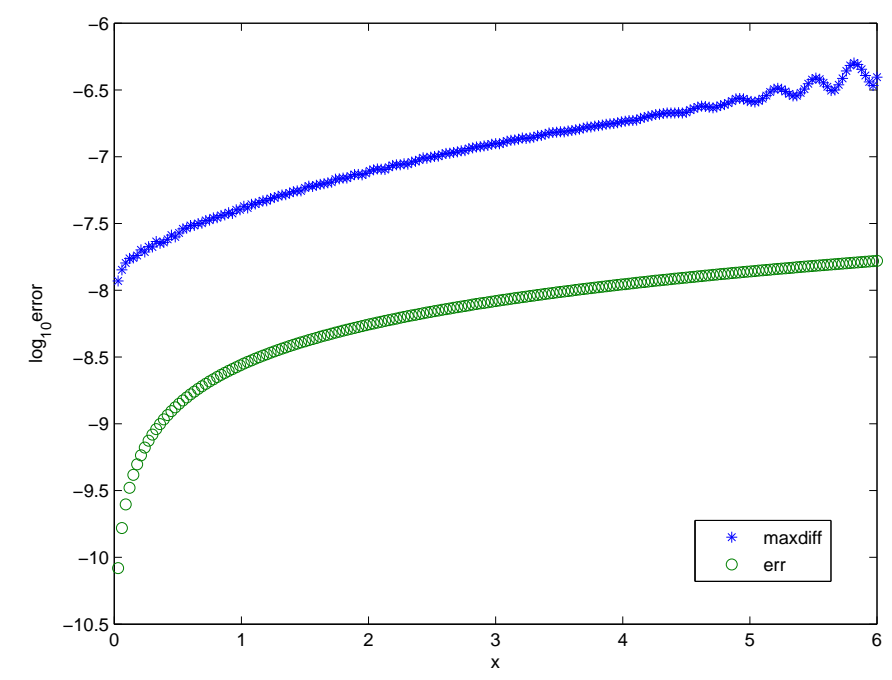

FiguRE 2. Numerical errors for the time evolution of 1-soliton initial data: $L^{\infty}$ norm of the difference between exact and numerical solution (maxdiff) and deviation from mass conservation (err).

An alternative test of the numerical precision is provided by conserved quantities. Due to unavoidable numerical errors such quantities will be numerically a function of time. Typically the energy (2.3) is a convenient quantity in this context. However the term $\partial_{x}^{-1} \partial_{y} u$ in general will have the same analytical properties as KP solutions not subject to the constraint (3.4), namely a cusp along the $x$-axis. Since we use spectral methods, this non-analyticity of the integrand in (2.3) would lead to numerical errors, which however are mainly due to the way the integral in (2.3) is evaluated, and not to the numerical error in $u(t)$ itself.

\footnotetext{
${ }^{1}$ MATLAB works internally with a precision of $10^{-16}$. Due to rounding errors machine precision is generally limited to the order of $10^{-14}$.
} 
Therefore we shall instead consider the mass (2.2), which is also conserved in time, but which admits an integrand with better regularity properties than the energy. Since mass conservation is not implemented, it provides a strong test for the accuracy of the code. We define the corresponding numerical error function by

$$
\operatorname{err}(t):=1-\frac{\mathrm{M}[u(t)]}{\mathrm{M}\left[u_{\mathrm{I}}\right]},
$$

where $\mathrm{M}[u(t)]$ is the numerically calculated mass which is obtained via FFT (in both spatial coordinates) of the integrand in (2.2) at each time step. For the example of the 1-soliton, this function is shown in Fig. 2 It can be seen that the error obtained via the integral quantity is typically one order of magnitude higher than the maximal local difference of the exact and the numerical solution. In cases where no exact solution is known, we shall use mass conservation as an indicator of the precision of the numerics. Consequently, the number of modes and the time steps will always be chosen in a way such that the value of the function $\operatorname{err}(t)$ is at least one order of magnitude lower than the precision of the numerical solution we are aiming at.

As an example for the instability of the above given line-soliton in the case of the KP-I equation, we consider, as in 34, a strongly perturbed line-soliton of (1.1) with initial data

$$
u_{\mathrm{I}}(x, y)=\frac{12}{\cosh ^{2}\left(x-x_{0}+\delta \cos (0.2 y)\right)} .
$$

In Fig. 3 we show the growth of the perturbation with time and the formation of lump solitons for $\delta=0.4, L_{x}=12, L_{y}=10, \Delta t=1.33 \times 10^{-4}$ and $N_{x}=N_{y}=512$. Due to the algebraic decay of the lump solitons for $|x|,|y| \rightarrow \infty$, relative mass conservation is of the order of $10^{-3.5}$ in this case. This is the precision with which the code propagates lump solitons for the given parameters.

Remark 3.2. Notice that the formation of power-law tails in the time evolution of initial data with compact support will lead to a Gibbs phenomenon at the boundaries in our spatially periodic setting. These effects are small for sufficiently large periods, but clearly noticeable. Thus in the examples studied here, relative mass conservation will be of the order of $10^{-7}$, even for very small times, whereas in the corresponding computations for the $\mathrm{KdV}$ equation it is of the order of machine precision [16]. On the other hand, on the longer time scales considered here (when studying the rapid modulated oscillations we are interested in), it is still possible to achieve relative mass conservations of the order of $10^{-4}$. The same holds for the propagation of lump solitons such as (2.5), where the slow (algebraic) decay as $|x|,|y| \rightarrow \infty$ again leads to Gibbs phenomena at the boundaries. Nonetheless our scheme is able to propagate lump solitons on standard computers with relative mass conservations of the order of $10^{-4}$ as the code in 12 .

\section{Modulation theORY FOR SMALl AMPLitUde SOLUtions}

Since the small dispersion limit $\epsilon \rightarrow 0$ of (1.1) is only poorly understood, we first consider the analogous problem for solutions which are asymptotically small, i.e. of order $\mathcal{O}(\epsilon)$. To this end we perform a (formal) multiple scales expansion, similar to those given in 36, 46, for such kind of solutions. Since the quadratic nonlinearity in this case is of lower order, we expect that the appearing fast oscillations can be described by a rather simple ansatz. More precisely they will be purely $x$-dependent. 

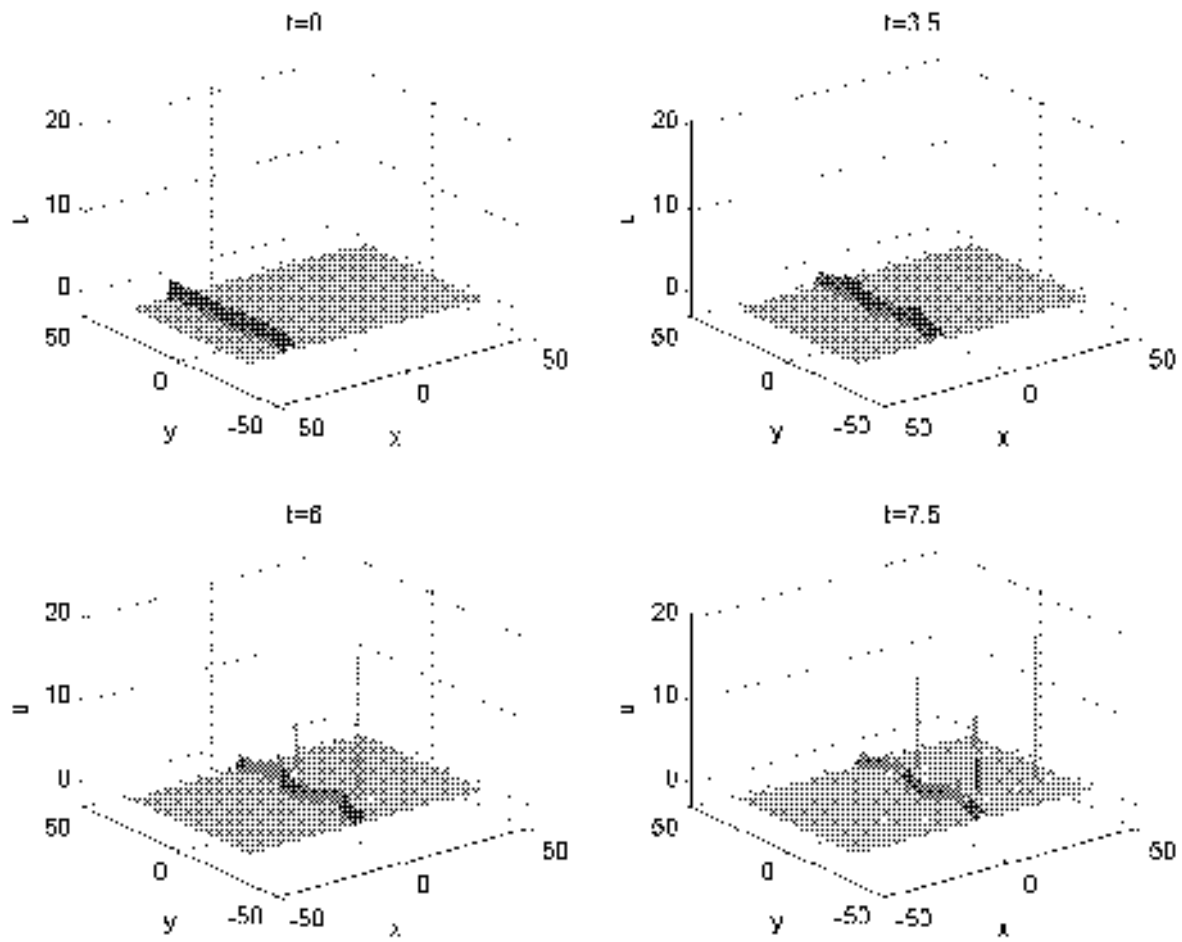

FiguRE 3. Time evolution of a perturbed line-soliton, growth of the perturbation and formation of lump solitons in the KP-I equation.

Namely, via the so-called Airy equation with plane wave initial data, i.e.

$$
\partial_{t} v+\partial_{x x x} v=0,\left.\quad v\right|_{t=0}=\mathrm{e}^{\mathrm{i} \eta x / \epsilon} .
$$

Note that this yields an asymptotic description which does not involve fast oscillations in the $y$-variable, $c f$. below. This is certainly a simplification which nevertheless is justified by the computations given in Section 2 for the linear equation and by our numerical results.

4.1. Multiple scales expansion. To be more precise, let us consider the scaled KP equation (1.1), assuming that its solutions admit an asymptotic expansion of the form

$$
u(t, x, y) \sim \epsilon u_{0}(t, x, y)+\epsilon^{2} u_{1}(t, x, y)+\mathcal{O}\left(\epsilon^{3}\right), \quad \epsilon \ll 1 .
$$

As usual in multiple scales expansion methods, we consider

$$
u_{j}=u_{j}(\tau, t, x, y, X, Y, T), \quad \forall j \in \mathbb{N},
$$

introducing again the fast variables $X=x / \epsilon, Y=y / \epsilon, T=t / \epsilon$, as well as the additional time-scale $\tau=\epsilon t$. Moreover we assume $u_{j}$ to be periodic w.r.t to $X, Y, T$. Note however that in the initial data (4.1) we do not take into account the fast scale $Y=y / \epsilon$. Thus we shall neglect this dependence in the following and one easily checks that this is consistent with our asymptotic expansion up to any order in $\epsilon$. 
Also note that we have to take into account additionally the (slowly varying) time scale $\tau=\epsilon t$ in order to balance the nonlinearity with the term $\partial_{x} \partial_{t} u$.

Equating powers of $\epsilon$ in our expansion, we find that the leading order term $u_{0}$ is given by

$$
u_{0}(t, x, y)=\psi_{0}\left(\epsilon t, x+3 \eta^{2} t, y\right) \mathrm{e}^{\mathrm{i}(\eta x+\omega(\eta) t) / \epsilon}+\text { c.c., } \quad \omega(\eta)=\eta^{3},
$$

for any given $\eta \in \mathbb{R}, \eta \neq 0$, where $\psi_{0}(t, \xi(t, x), y) \in \mathbb{C}$ and "c.c." refers to the complex conjugate. The fast oscillations are henceforth described by plane waves propagating according to $\omega(\eta)=\eta^{3}$, the dispersion relation of the Airy equation. Moreover, in (4.4), we need to take into account a drift defined by

$$
\xi(t, x):=x+3 \eta^{2} t
$$

i.e. we need to perform a change of variables into a reference frame moving at group velocity $v_{\mathrm{g}}=3 \eta^{2}$. Thus, on the so-called ballistic scales $(t, x)=(\epsilon T, \epsilon X)$, the slowly varying amplitude satisfies the transport equation

$$
\partial_{t} \psi_{0}-3 \eta^{2} \partial_{x} \psi_{0}=0
$$

which is obtained from the solvability condition for terms of order $\mathcal{O}(\epsilon)$.

Consequently one finds that the fast scale dynamics in the first order corrector $u_{1}$ is determined via

$$
\partial_{X}\left(\partial_{T}+\partial_{X X X}\right) u_{1}=-\frac{1}{2} \partial_{X X}\left(\psi_{0}^{2}(\tau, \xi(t, x), y) \mathrm{e}^{2 \mathrm{i}(\eta X+\omega(\eta) T)}+\text { c.c. }\right)
$$

and thus $u_{1}$ is given by

$$
\begin{aligned}
u_{1}(t, x, y)= & \frac{1}{6 \eta^{2}} \psi_{0}^{2}(\tau, \xi(t, x), y) \mathrm{e}^{2 \mathrm{i}(\eta X+\omega(\eta) T)}+\psi_{1}(\tau, \xi(t, x), y) \mathrm{e}^{\mathrm{i}(\eta X+\omega(\eta) T)} \\
& + \text { c.c. }+\left.\phi(\tau, \xi(t, x), y)\right|_{\tau=\epsilon t, X=x / \epsilon, T=t / \epsilon}
\end{aligned}
$$

where $\phi$ can now be interpreted as a mean field generated by $\psi_{0}$. From the mathematical point of view it is necessary to take into account this non-oscillatory field to balance the terms generated via the quadratic nonlinearity. We eventually find that $\psi_{0}$ and $\phi$ solve a coupled system of Davey-Stewartson type (DS). More precisely we get

$$
\left\{\begin{aligned}
\mathrm{i} \partial_{\tau} \psi_{0}-3 \eta \partial_{\xi \xi} \psi_{0}+\frac{\lambda}{\eta} \partial_{y y} \psi_{0}-\left(\frac{1}{6 \eta}\left|\psi_{0}\right|^{2}+\eta \phi\right) \psi_{0} & =0 \\
3 \eta^{2} \partial_{\xi \xi} \phi+\lambda \partial_{y y} \phi+\partial_{\xi \xi}\left|\psi_{0}\right|^{2} & =0 .
\end{aligned}\right.
$$

This mean field system describes the dispersive effects which are visible on timescales of order $\mathcal{O}(\epsilon t)$. Analogous to the KP equation, the DS system represents a completely integrable model in $d=2$ spatial dimensions. The case where, for $\eta>0, \lambda=+1$, is referred to as the "hyperbolic-elliptic" case, cf. [36], whereas for $\lambda=-1$ we are in the so-called "elliptic-hyperbolic" situation. Here we shall only focus on the former case, induced by the KP-II model, as the latter requires a special numerical treatment due to the appearing wave-type operator in the second equation of (4.8). Thus we require, that the mean field behaves like

$$
\phi(\tau, \xi, y) \rightarrow 0, \quad \text { as }|\xi|,|y| \rightarrow \infty .
$$

Remark 4.1. An analogous asymptotic expansion for the KdV equation yields a cubic nonlinear Schrödinger equation, instead of the DS system, as has been rigorously proved in 35. We expect that a similar analytical strategy could also be applied in our case to rigorously establish the above given formal asymptotics. 
In the present work, the numerical algorithm used to solve (4.8) is analogous to the one used for the KP equation (for an alternative approach see [4]). More precisely, denote by $\widehat{\psi_{0}}\left(\tau, k_{\xi}, k_{y}\right)$ the Fourier transform of $\psi_{0}(\tau, \xi, y)$ and let $\widehat{\psi_{0}\left|\psi_{0}^{2}\right|}$ and $\widehat{\psi_{0} \phi}$ be the Fourier transforms of $\psi_{0}\left|\psi_{0}^{2}\right|$ and $\psi_{0} \phi$, respectively. Thus we get for the system (4.8)

$$
\left\{\begin{array}{r}
\mathrm{i} \partial_{\tau} \widehat{\psi_{0}}+\left(3 \eta k_{\xi}^{2}-\frac{\lambda}{\eta} k_{y}^{2}\right) \widehat{\psi_{0}}-\frac{1}{6 \eta} \widehat{\psi_{0}\left|\psi_{0}^{2}\right|}-\eta \widehat{\psi_{0} \phi}=0, \\
\widehat{\phi}=-\frac{\eta k_{\xi}^{2}}{3 \eta^{2} k_{\xi}^{2}+\lambda k_{y}^{2}} \widehat{\left|\psi_{0}^{2}\right|},
\end{array}\right.
$$

with $\eta>0$. Again we shall use an integrating factor to avoid a stiff part in the first equation of (4.10), i.e.

$$
\partial_{\tau}\left(\mathrm{e}^{-\mathrm{i} \tau\left(3 \eta k_{\xi}^{2}-\lambda k_{y}^{2} / \eta\right)} \widehat{\psi_{0}}\right)+\mathrm{i} \mathrm{e}^{-\mathrm{i} \tau\left(3 \eta k_{\xi}^{2}-\lambda k_{y}^{2} / \eta\right)}\left(\frac{1}{6 \eta} \widehat{\psi_{0}\left|\psi_{0}^{2}\right|}+\eta \widehat{\psi_{0} \phi}\right)=0 .
$$

The time integration will be carried out as before with a fourth order Runge-Kutta method. Accuracy of the code will be checked by conservation of the wave energy

$$
\mathrm{N}\left[\psi_{0}(\tau)\right]:=\int_{\mathbb{R}^{2}}\left|\psi_{0}(\tau, \xi, y)\right|^{2} \mathrm{~d} \xi \mathrm{d} y,
$$

which is preserved in time for the DS system (4.8).

4.2. Numerical examples. To study a concrete example we consider, for $\eta=1$, real-valued initial data to the DS system (4.8) in the following form

$$
\left.\psi_{0}\right|_{t=0} \equiv \psi_{\mathrm{I}}(x, y)=-\partial_{x} \operatorname{sech}^{2}(R), \quad R:=\sqrt{x^{2}+y^{2}} .
$$

The choice for these initial data is motivated by the fact that they are smooth, localized in $x, y$, and that they could, in principle, also serve as initial data for the KP equation (1.1), since they satisfy the constraint (3.4).

We then solve the DS system (4.8) with initial data (4.13) for $t \in[0,0.4]$ with $N_{x}=$ $N_{y}=512$ and $\Delta t=2 \times 10^{-3}$. The wave energy is conserved in this computation up to the order of machine precision (the error is smaller than $10^{-13}$ ). We show

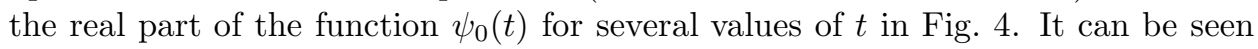
that the initially localized pulse spreads and exhibits oscillations, both mainly in $x$-direction. For longer times, the modulation of the DS solution becomes more pronounced as can be seen in Fig. [5 Notice that the real and imaginary part of $\psi_{0}(t)$ have almost the same envelope, they mainly differ by a phase shift. This is more obvious by considering the absolute value of $\psi_{0}$ in Fig. [6 which virtually shows no modulations.

Corresponding to (4.13), the KP-II equation is then solved with initial data

$$
u_{\mathrm{I}}(x, y)=2 \epsilon \psi_{\mathrm{I}}(R) \cos \left(\frac{x}{\epsilon}\right), \quad R=\sqrt{x^{2}+y^{2}} .
$$

Note however, that these initial data do not satisfy the constraint (3.4). To enforce (3.4) we numerically compute the Fourier transform of $u_{\mathrm{I}}$ and set the Fourier coefficients of all terms corresponding to $k_{x}=0$ in these data equal to zero. This can be justified by the fact that in our examples, where we take $\epsilon=0.1$ and smaller, these Fourier coefficients are only of the order $10^{-14}$, i.e. of the order of the rounding error. Thus, within numerical precision, we can directly use these initial data, which are now adapted to both, the asymptotic expansion and the integral constraint (3.4). For the precise parameters used in the computations of the KP equation, for times between 0 and 4 , see table 1 

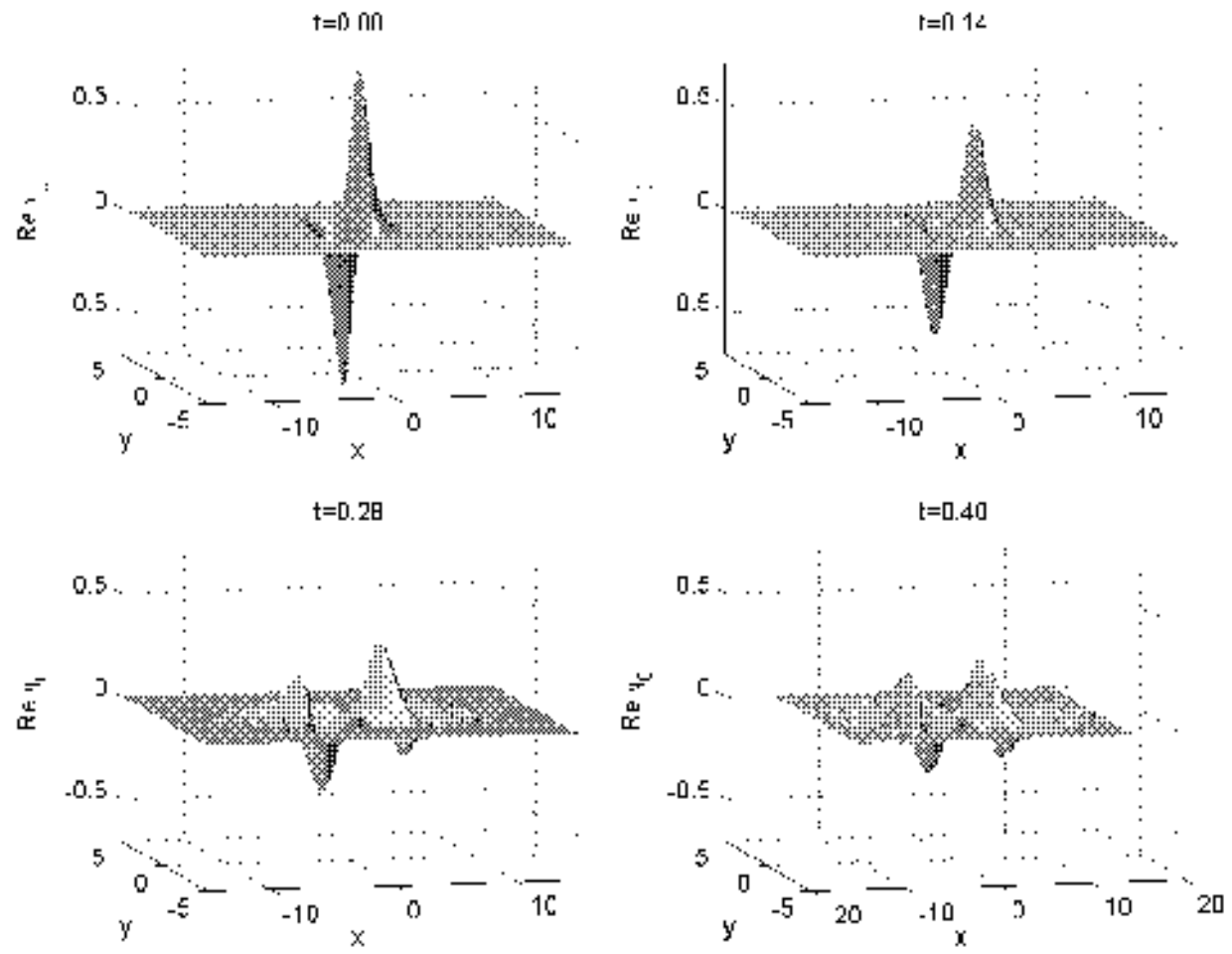

Figure 4. Time evolution of $\operatorname{Re} \psi_{0}(t)$, solution to the DS system with initial data (4.13), shown for several values of $t \geq 0$.
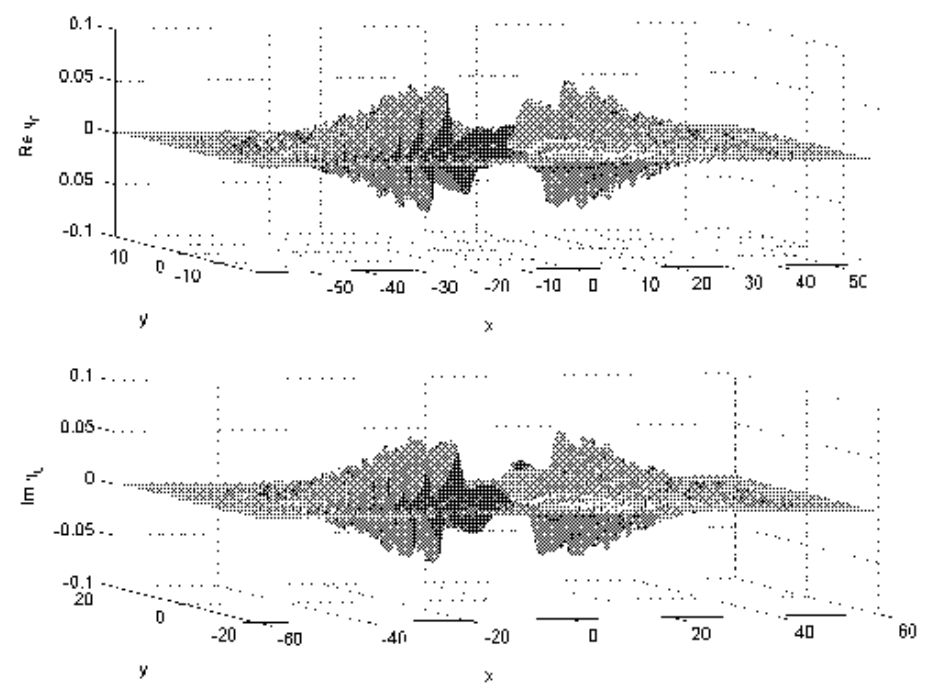

Figure 5. $\operatorname{Re} \psi_{0}(t)$ and $\operatorname{Im} \psi_{0}(t)$, obtained from the initial data (4.13), at time $t=2$. 


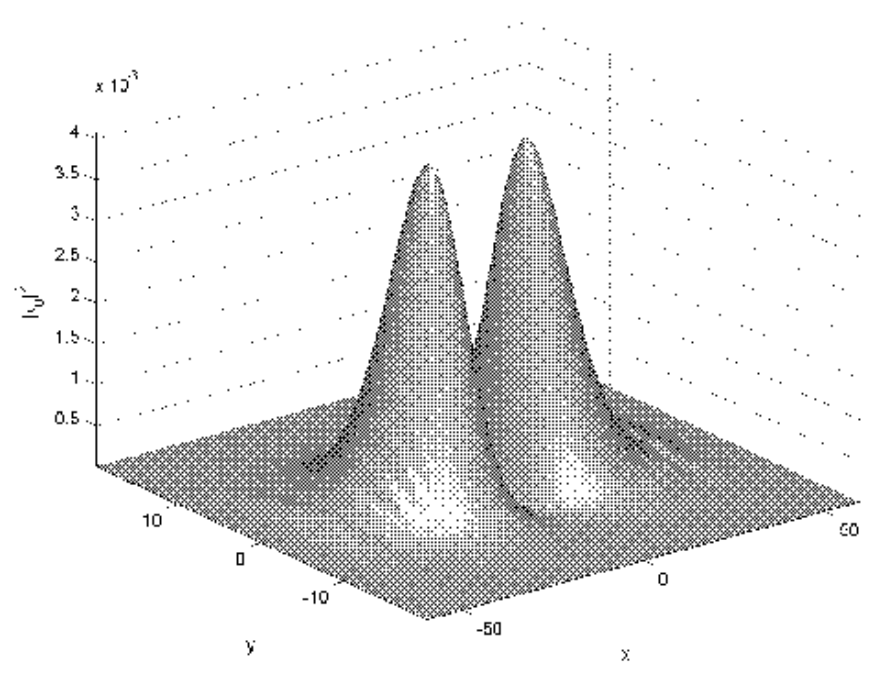

Figure 6. $\left|\psi_{0}(t)\right|^{2}$, obtained from the initial data (4.13), at time $t=2$.

\begin{tabular}{|c|c|c|c|c|c|c|}
\hline$-\log _{10} \epsilon$ & $\log _{2} N_{x}$ & $\log _{2} N_{y}$ & $\Delta t$ & $L_{x}$ & $L_{y}$ & $\log _{10} e r r$ \\
\hline 1 & 10 & 7 & $8 \times 10^{-5}$ & 10 & 10 & 4.98 \\
\hline 1.25 & 11 & 7 & $8 \times 10^{-5}$ & 10 & 10 & 4.23 \\
\hline 1.5 & 12 & 7 & $6.67 \times 10^{-5}$ & 10 & 10 & 4.21 \\
\hline 1.75 & 12 & 7 & $8 \times 10^{-5}$ & 5 & 5 & 4.07 \\
\hline 2 & 12 & 7 & $6.67 \times 10^{-5}$ & 5 & 5 & 4.64 \\
\hline
\end{tabular}

TABLE 1. Parameters in the computation of the KP solutions

In Fig. 7 we plot the solution of the KP equation for $\epsilon=0.1$ for several values of $t$. As expected the pulse travels roughly with the velocity $v_{\mathrm{g}}=3$ (notice that the shown region in the plots is co-moving as can be seen from the $x$-scales) and the initially localized pulse spreads mainly in $x$-direction. Since we only consider small amplitudes here, the characteristic KP tails are hardly visible on the studied timescales. Thus the periodic boundary conditions do not influence the model.

The above initial data are of the required form for the asymptotic expansion (4.2) in leading order of $\epsilon$. In principle one could try to obtain a refined asymptotics, including higher order corrector terms. This however yields the highly nontrivial problem of satisfying the KP constraint (3.4) up to sufficient high order. More precisely if one takes into account also the first order corrector (4.7), then, since $\widehat{\phi}(t)$ is proportional to the Fourier transform of $\left|\psi_{0}(t)\right|^{2}$, the coefficients for $k_{x}=0$ are of the order $\mathcal{O}\left(\epsilon^{2}\right)$. In order to satisfy the constraint also up to $\mathcal{O}\left(\epsilon^{2}\right)$ errors, it consequently would be necessary either to choose $\psi_{2}(0, x, y)$ in an appropriate way, or to consider a function $\psi_{\mathrm{I}}(x, y)$ which is subject to a nonlinear integral constraint following from (3.4). In the following we shall simply neglect higher order corrector terms in the initial data as we only aim (numerically) for the leading order asymptotic description. 

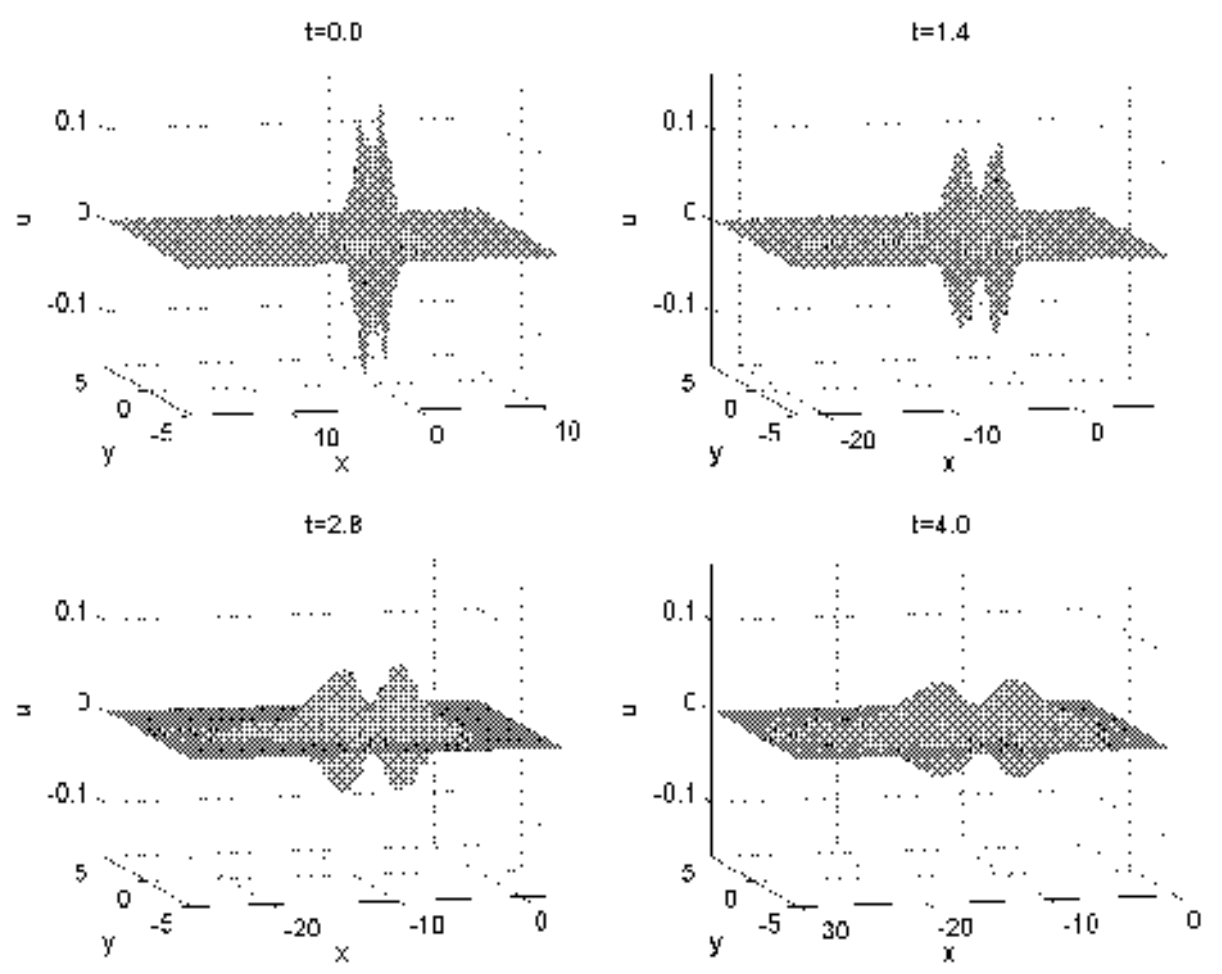

Figure 7. Time evolution of the initial data (4.14), governed by the KP-II equation with $\epsilon=0.1$, shown for several values of $t \geq 0$ and shifted by $x \rightarrow x-12$.

The above given expansion indicates that the solution to the DS system (4.8), with initial data $\psi_{\mathrm{I}}$, should approximate, via (4.2) and (4.4), the corresponding KP solution up to errors of order $\mathcal{O}\left(\epsilon^{\alpha}\right)$, for some $\alpha>1$, on any finite time-interval. In Fig. [ we plot the approximating solution

$$
u_{\text {app }}(t, x, y)=2 \epsilon \operatorname{Re}\left(\psi(\epsilon t, \xi(t, x), y) \mathrm{e}^{\mathrm{i}(x+t) / \epsilon}\right),
$$

for several values of $t$. It can be seen that this asymptotic solution gives the expected description of the KP solution, which is even better visible in Fig. 9 where we have plotted the two solutions for $t=4$ and $y=0$ in one frame. The difference of these two solutions for $t=4$ can be seen for $y=0$ in Fig. 10 and in the whole $(x, y)$-plane in Fig. 11

Remark 4.2. Notice the difference between the KP solution and the asymptotic solution for different signs of the co-moving coordinate $\xi(t, x)$. We chose initial data which are odd in $x$. Obviously the KP equation does not conserve the parity of the initial data, whereas the DS system does. In our example the asymptotic solution is, up to the leading order in $\epsilon$, an odd function in $x$, whereas this is no longer true for the KP solution. Higher order terms in the asymptotic solution, which we neglected here, will break this symmetry.

For smaller values of $\epsilon$ the approximation gets better, but the numerical resolution of the high frequencies becomes increasingly more difficult. The main problem is 

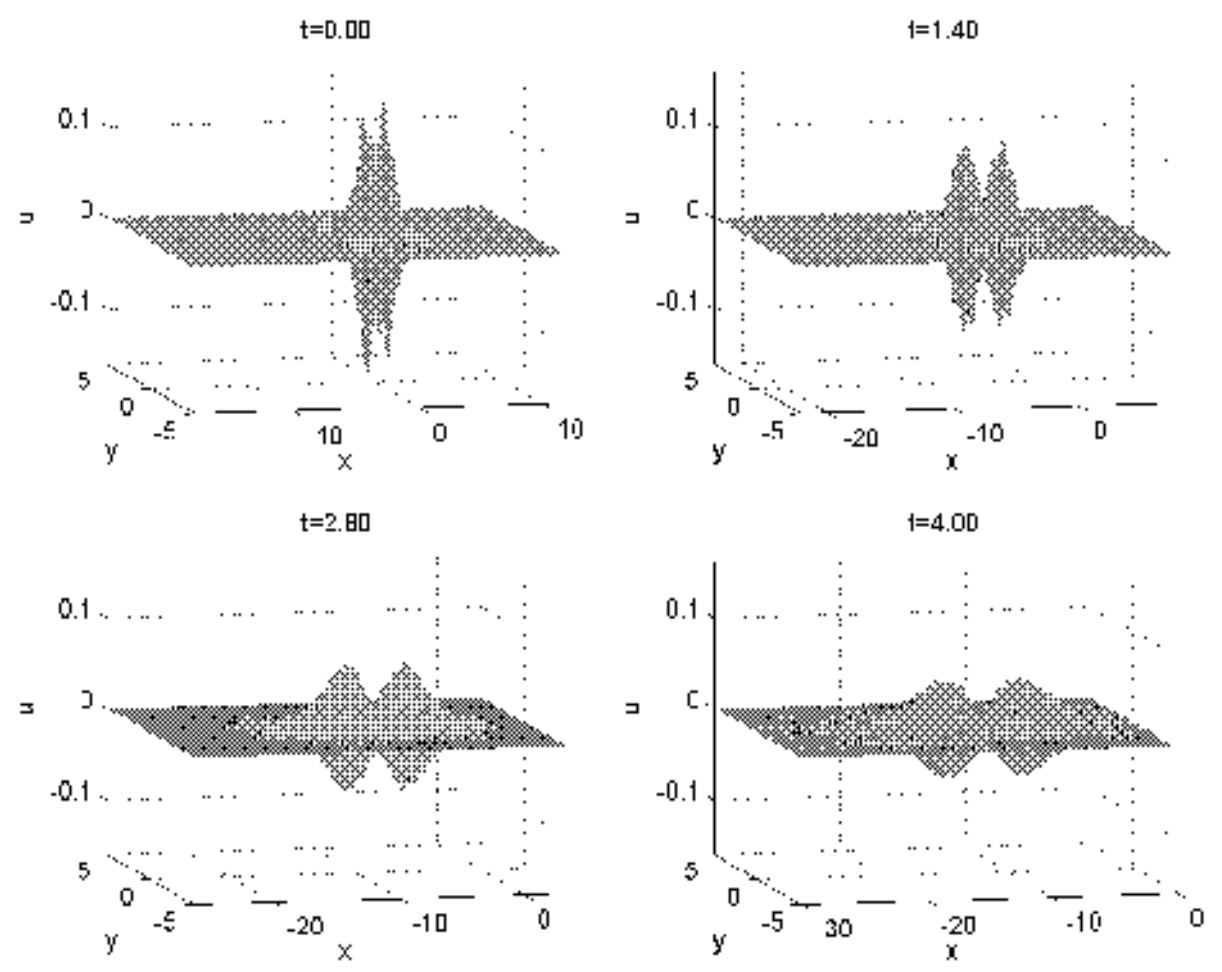

Figure 8. The asymptotic solution $u_{\text {app }}(t, x, y)=$ $2 \epsilon \operatorname{Re}\left(\psi(\epsilon t, \xi(t, x), y) \mathrm{e}^{\mathrm{i}(x+t) / \epsilon}\right)$ approximating the true KP-II solution of Fig. [7 shown for several values of $t \geq 0$.

that the DS system approximates the KP solution only on the time scale $\epsilon$. Thus in order to see the effects of the modulations due to the DS system within the KP solution one would need to solve the KP equation for extremely long times if $\epsilon$ is chosen very small. This is of course numerically rather expensive, in particular in our $2+1$ dimensional case. We will therefore limit our analysis to the range $0.01 \leq$ $\epsilon \leq 0.1$. The above given example however clearly illustrates the applicability of the asymptotic expansion. For $\epsilon=0.01$ the point wise difference between the KP solution and the asymptotic solution is, as expected, at most of the order $10^{-4}$ and thus barely one order of magnitude higher than the numerical error, cf. Fig. 12 To get more insight we plot the $L^{\infty}\left(\mathbb{R}^{2}\right)$ norm of the difference, denoted by $\Delta_{\infty}(t)$, in dependence of $\epsilon$ in Fig. [13. This error is found to be monotonically increasing in time and furthermore it decreases roughly like $\epsilon^{9 / 4}$. Indeed the data obtained at the last time-step $t=4$, can be fitted by a straight line $-\log _{10} \Delta_{\infty}=-a \log _{10} \epsilon+b$ with $a=2.27$ and $b=-0.58$. The correlation coefficient is then found to be $r=0.999$, the standard error for $a$ is $\sigma_{a}=0.08$.

To define an integral quantity $\Delta_{2}(t)$ for the error between the true KP solution and its (leading order) asymptotic description 4.15), we also consider the $L^{2}\left(\mathbb{R}^{2}\right)$ norm of the difference. Indeed this is the most widely used definition of an asymptotic error in such singular limiting regimes, cf. 35] for the analogous definition in the $\mathrm{KdV}$ case. Thus we integrate the square of the difference of these solutions over the domain of definition via an FFT (in both variables) and normalize this quantity 


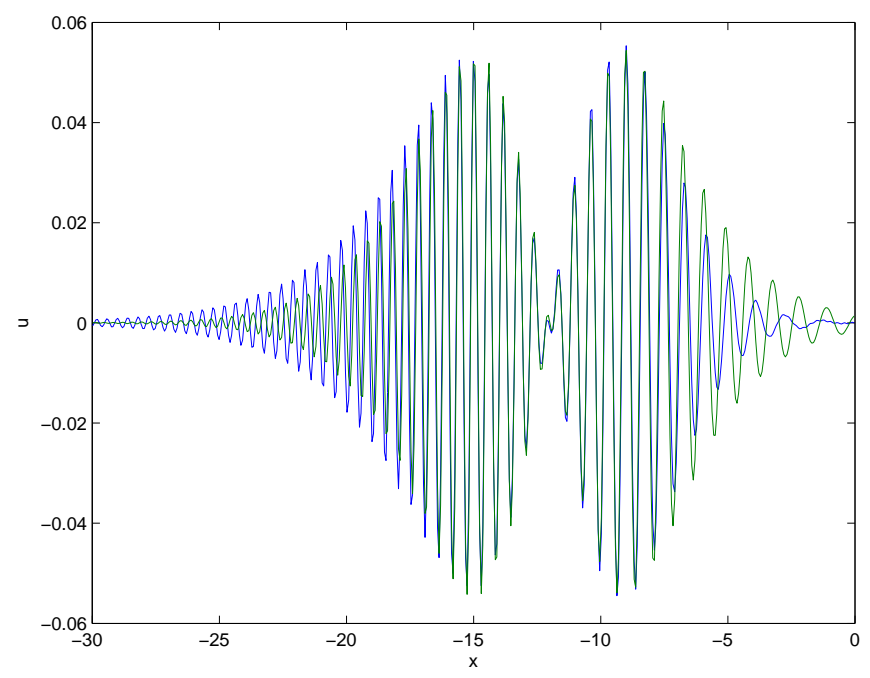

Figure 9. Solution to the KP-II equation with initial data (4.14) (blue) and the corresponding asymptotic solution (green) for $y=0$, $t=4$, and $\epsilon=0.1$.

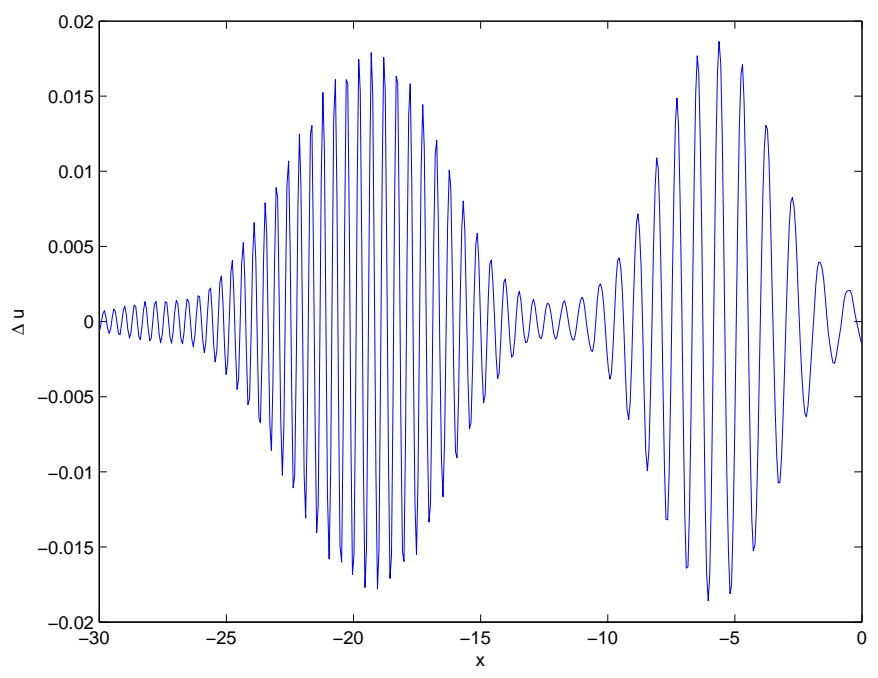

FIGURE 10. Difference of the solution to the KP-II equation with initial data (4.14) and the corresponding asymptotic solution for $y=0, t=4$, and $\epsilon=0.1$.

dividing it by the area of the fundamental domain, i.e.

$$
\Delta_{2}(t):=\frac{1}{2 \pi \sqrt{L_{x} L_{y}}}\left(\int_{-\pi L_{x}}^{\pi L_{x}} \int_{-\pi L_{y}}^{\pi L_{y}}\left(u(t, x, y)-u_{\text {app }}(t, x, y)\right)^{2} \mathrm{~d} x \mathrm{~d} y\right)^{1 / 2} .
$$




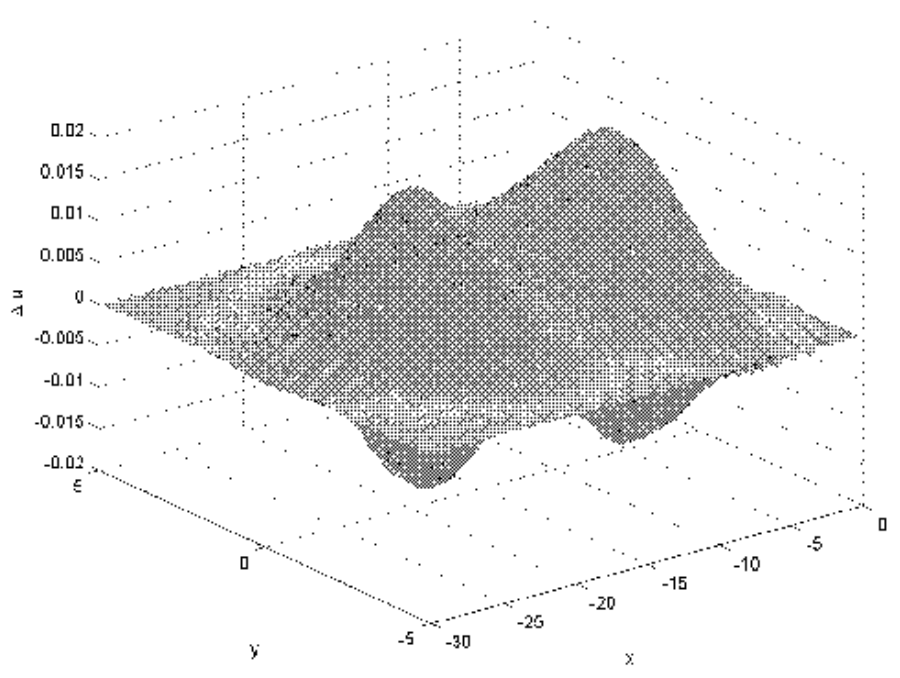

Figure 11. Difference of the solution to the KP-II equation with initial data (4.14) and the corresponding asymptotic solution for $t=4$ and $\epsilon=0.1$.

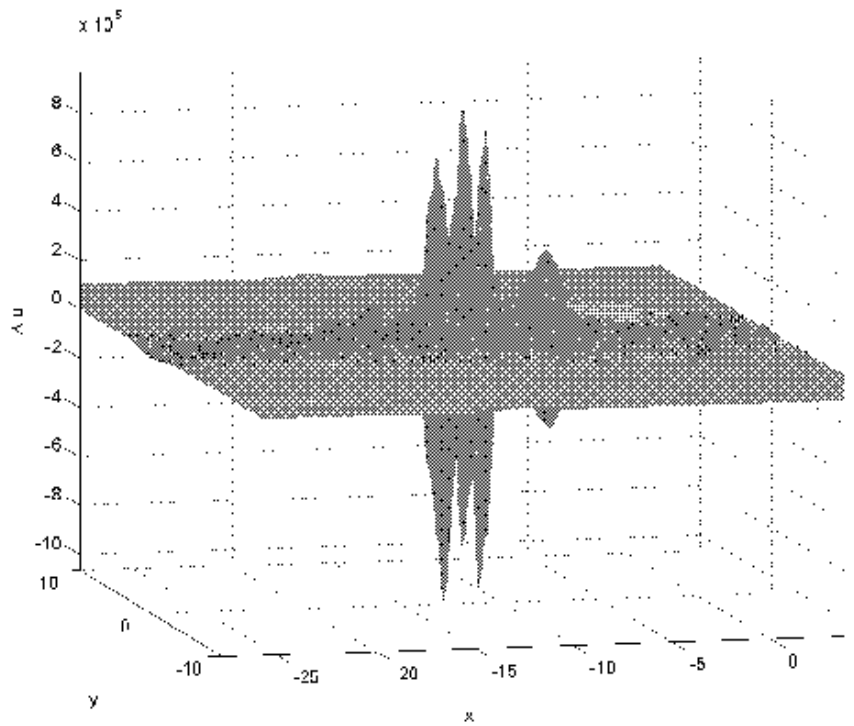

Figure 12. Difference of the solution to the KP-II equation with initial data (4.14) and the corresponding asymptotic solution for $t=4$, and $\epsilon=10^{-2}$.

We find that $\Delta_{2}(t)$ again increases monotonically in time. For $t=4$ we get the values of $\Delta_{2}$ shown in Fig. 14. By linear regression the data can again be fitted by a straight line $-\log _{10} \Delta_{2}=-a \log _{10} \epsilon+b$, with $a=2.48$ and $b=0.53$. The correlation coefficient in this case is $r=0.997$ and the standard error for $a$ is $\sigma_{a}=0.17$. 


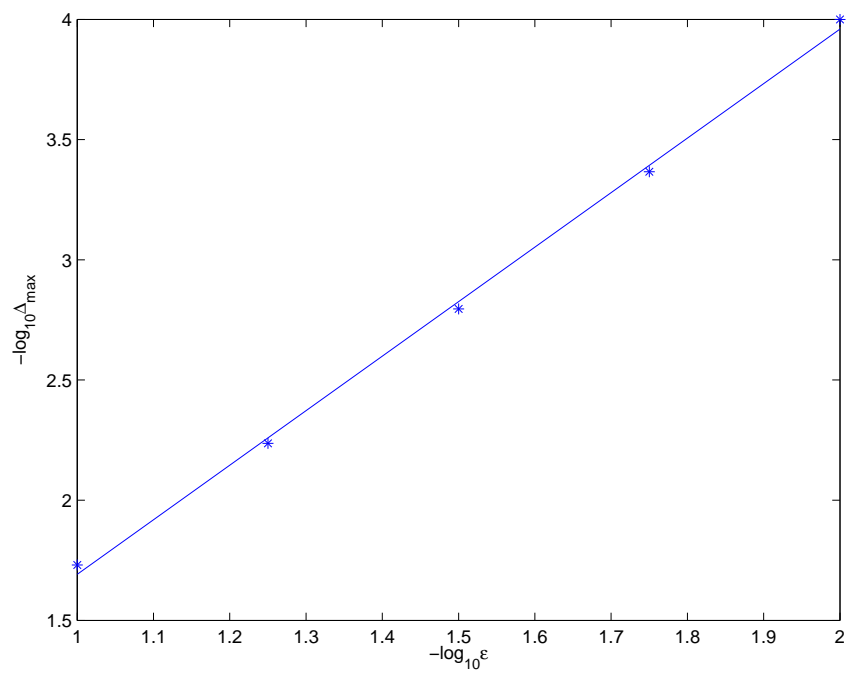

Figure 13. Error $\Delta_{\infty}$ for several values of $\epsilon$. The data can be fitted by least square analysis with a straight line $-\log _{10} \Delta_{\infty}=$ $-a \log _{10} \epsilon+b$ with $a=2.27$ and $b=-0.58$. The correlation coefficient is $r=0.999$, the standard error for $a$ is $\sigma_{a}=0.08$.

Remark 4.3. It is not surprising that $\Delta_{2}$ decreases faster with $\epsilon$ than $\Delta_{\infty}$ since not only do the amplitudes shrink with $\epsilon$, but also the pulse is more localized in $x$ and $y$. Thus a smaller integral error is to be expected.

In other words we get that numerically the $L^{2}\left(\mathbb{R}^{2}\right)$ difference between the true KP solution and its asymptotic description is approximately of the order $\mathcal{O}\left(\epsilon^{5 / 2}\right)$. This is remarkable since it fits with the analytical results of [35] where the analogous limit from KdV to the cubic nonlinear Schrödinger equation is considered.

Remark 4.4. Note however, that for a comparison of our asymptotic description (4.4) with the one given in [35] one has to take into account a rescaling of the spatial scales on which the modulation amplitudes varies. Thus rescaling $x$ and $y$ (the latter scale is of course not present in [35]; one has to plug this in "by hand") such that the two descriptions match each other, one realizes that an additional factor $\epsilon$ has to be taken into account in order to conserve the $L^{2}\left(\mathbb{R}^{2}\right)$ norm of the solutions. In summary one checks that Theorem 1 in 35 yields an asymptotic error of the order $\mathcal{O}\left(\epsilon^{5 / 2}\right)$, i.e. exactly as in our case.

\section{Small dispersion KP solutions with amplitudes of order $\mathcal{O}(1)$}

In this section we will study numerically solutions to the KP equation in the regime of small dispersion $\epsilon \ll 1$. In contrast to Section 4 though we shall not restrict ourselves to small amplitude solutions. Rather we shall consider smooth initial data with an amplitude of order $\mathcal{O}(1)$, as $\epsilon \rightarrow 0$. To this end let us first discuss briefly the resulting (formal) limiting equation (1.8) and its numerical implementation in the next subsection. We shall then investigate a concrete example with initial data of the form

$$
u_{\mathrm{I}}(x, y)=-6 \partial_{x} \operatorname{sech}^{2}(R), \quad R=\sqrt{x^{2}+y^{2}},
$$




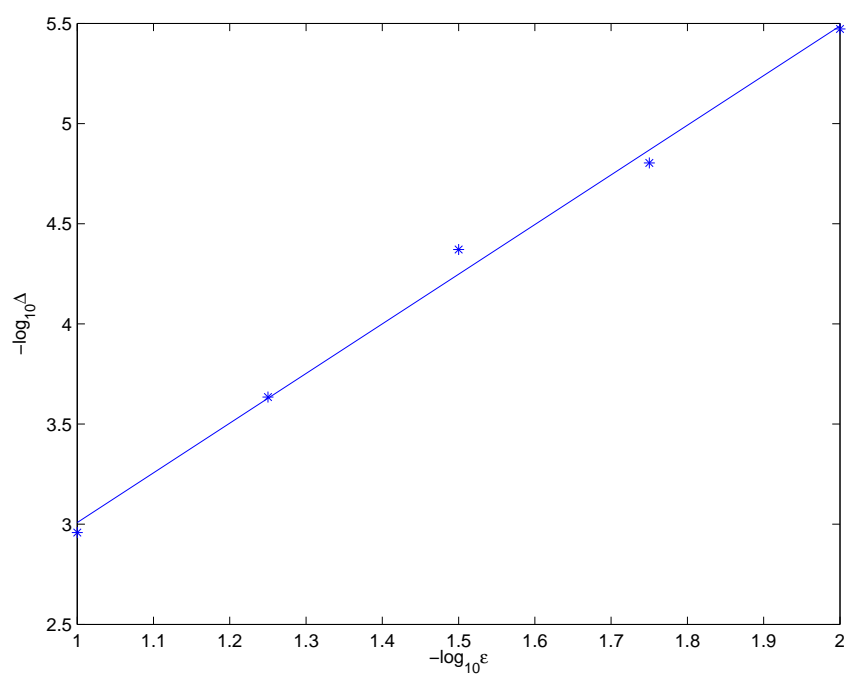

Figure 14. Error $\Delta_{2}$ as defined in 4.16 for several values of $\epsilon$. The data can be fitted by least square analysis with a straight line $-\log _{10} \Delta_{2}=-a \log _{10} \epsilon+b$ with $a=2.48$ and $b=0.53$. The correlation coefficient is $r=0.997$, the standard error for $a$ is $\sigma_{a}=0.17$.

as can be seen in Fig. 15] Note that these data satisfy the constraint (2.10). The

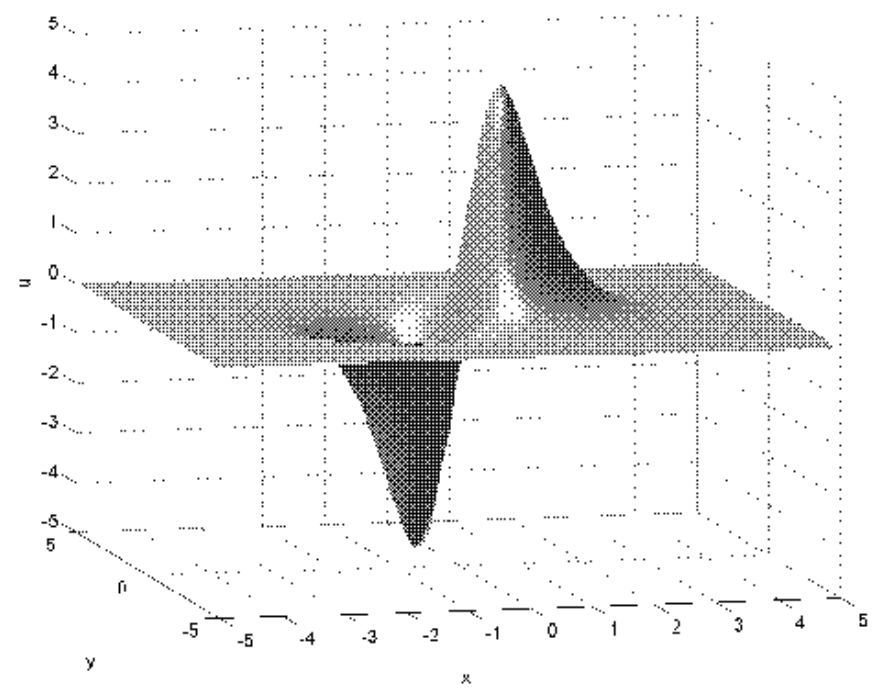

Figure 15. Initial conditions for the KP equation.

factor 6 in (5.1) is included in order to be able to compare our results with those given in 16 for the $\mathrm{KdV}$ equation, where a different definition of the amplitude $u(t)$ was used (the $u(t)$ there corresponds to $6 u(t)$ here). With the introduction of 
the factor 6 we expect the same dynamical time scales in the $\mathrm{KdV}$ sector of (1.1) as in [16].

5.1. The dKP equation and its dissipative regularization. We do not expect the formal limiting dKP equation (1.8), i.e.

$$
\partial_{x}\left(\partial_{t} u+u \partial_{x} u\right)+\lambda \partial_{y y} u=0
$$

to be the correct description of the dispersionless limit for (1.1), at least not for all times $t \geq 0$. This believe stems from the closely related situation encountered in the inviscid Burgers, or Hopf equation, given by

$$
\partial_{t} u+u \partial_{x} u=0,\left.\quad u\right|_{t=0}=u_{\mathrm{I}}(x)
$$

Equation (5.2) can be seen as the (formal) dispersionless limit of the KdV equation. In general though this only holds for some finite time $0 \leq t_{c}<\infty$. More precisely, the description of the disperionless $\mathrm{KdV}$ limit via (5.2) fails, after the appearance of the first shock in the solution of (5.2). The corresponding break time is given by

$$
t_{c}=\min _{x_{0} \in \mathbb{R}}\left(-\frac{1}{\partial_{x} u_{\mathrm{I}}\left(x_{0}\right)}\right),
$$

which can be easily seen when solving (5.2) by the method of characteristics, yielding

$$
u(t, x)=u_{\mathrm{I}}\left(x_{0}\right), \quad x=u_{\mathrm{I}}\left(x_{0}\right) t+x_{0},
$$

the so-called Hopf solution. Consequently, we also expect in the case of the dKP equation that a shock will be formed after some finite time. (Clearly the behavior of the dKP equation is completely analogous to the Burger's case, if one considers $y$-independent solutions.) On the other hand, for small, but still finite, $\epsilon \ll 1$, this "dKP-shock" presumably will be smoothed out by rapid oscillations, similar to the $\mathrm{KdV}$ case, $c f$. the numerical examples below.

Remark 5.1. To convince ourselves that, apart from the KdV sector, the concept of shocks is really applicable in the dKP equation we remark that its principal part is given by

$$
P u:=\partial_{x t} u+u \partial_{x x} u+\lambda \partial_{y y} u .
$$

As one easily checks, the coefficient matrix in this partial differential operator $P$ admits three, distinct, real eigenvalues given by

$$
\mu_{1,2}=\frac{1}{2}\left(u \pm \sqrt{u^{2}+1}\right), \mu_{3}=\lambda .
$$

Thus the dKP equation indeed falls into the class of second order hyperbolic PDEs.c However the associated initial value problem as considered in this work, furnishes a characteristic Cauchy problem, a fact which has already been discussed in Subsection 2.2

To circumvent the problem of shock solutions in the formal limiting dKP model we shall use a dissipative regularization. More precisely we add a small dissipative term, in the form

$$
\partial_{x}\left(\partial_{t} u+u \partial_{x} u-\sigma \partial_{x x} u\right)+\lambda \partial_{y y} u=0, \quad \lambda= \pm 1 .
$$

with $0<\sigma \ll 1$ being some small real-valued parameter, such that $\sigma \sim \mathcal{O}(1)$, as $\epsilon \rightarrow 0$. Equation (5.7) can now be seen as the KP analog of the viscous Burgers equation, i.e.

$$
\partial_{t} u+u \partial_{x} u-\sigma \partial_{x x} u=0
$$


As in (3.2) we obtain that (5.7) can be solved via

$$
\partial_{t}\left(\mathrm{e}^{t\left(\mathrm{i} \lambda k_{y}^{2} /\left(k_{x}+\mathrm{i} \lambda 0\right)+\sigma k_{x}^{2}\right)} \widehat{u}\right)+\frac{\mathrm{i}}{2} k_{x} \mathrm{e}^{t\left(\mathrm{i} \lambda k_{y}^{2} /\left(k_{x}+\mathrm{i} \lambda 0\right)+\sigma k_{x}^{2}\right)} \widehat{u^{2}}=0 .
$$

Obviously equation (5.7) is no longer conservative, but the dissipative term will smooth out the shocks of the dKP equation. In other words, as $\sigma \rightarrow 0$, we expect the solution of (5.7) to tend to some kind of entropy solution.

Remark 5.2. Without the dissipative regularization, the numerical code would break down shortly before the formation of shocks, due to the appearing steep gradients which cause instabilities in our explicit time integration scheme. However, even with an implicit, unconditionally stable method, such as Crank-Nicholson, the numerical code would break down close to a shock. The reason for this is mainly due to the so-called aliasing error, i.e. a pollution of the spectral coefficients by high frequencies, see, e.g., 9]. Because of the nonlinearity in the KP equation, this high frequency noise yields severe problems near the gradient catastrophe. Even a de-aliasing, as used in, e.g., [16 for the KdV case, will not stabilize the code close to the breakup, since the aliasing error simply cannot be suppressed there.

Since the dKP equation is also completely integrable, many explicit solutions are known, cf. [13, 26, 27. In our study, though, we are interested in rather general initial data, where no closed form of the corresponding solutions is known. In the case (5.1) the corresponding KP-I solution $(\lambda=-1)$ is shown in Fig. 16] where $\sigma=0.01$. The computation was carried out with $N_{x}=4096, N_{y}=128$,

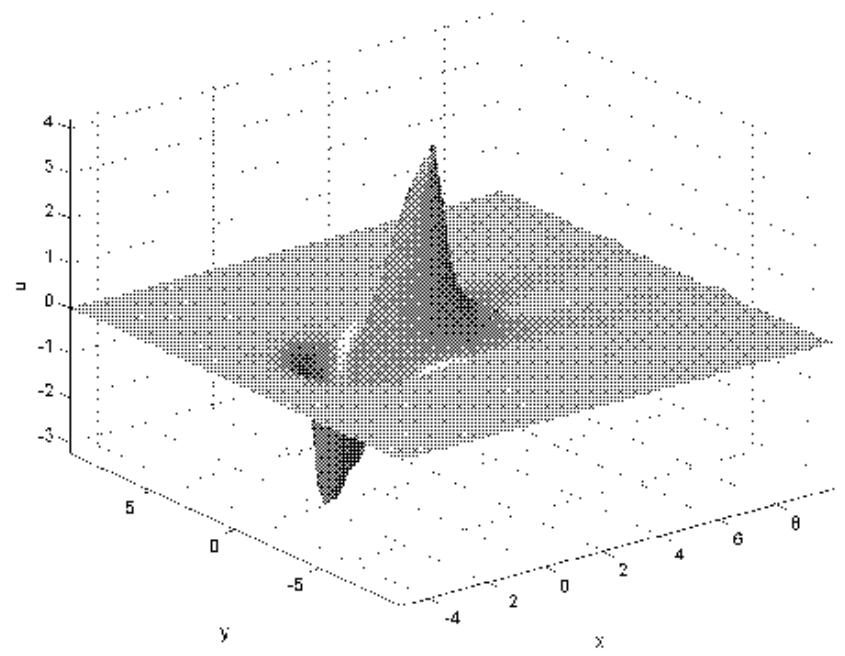

Figure 16. Solution to the regularized dKP-I equation with initial data (5.1) and $\sigma=0.01$, plotted at time $t=0.3$.

$L_{x}=L_{y}=10$ and $\Delta t=5 \times 10^{-5}$. Due to the dissipative term $\propto \sigma \partial_{x x} u$ the mass is no longer conserved, but in the given example the loss is only of the order of a few percent. Thus the shown solution should indeed be very close to a true shock solution of the dKP equation. Note that the tails, as $x \rightarrow \infty$, are clearly visible. Out focus here is now on the the region of steep gradients in the solutions to dKP. To this end it can be seen from Fig. [17 that the derivative $\partial_{x} u$ is always maximal 


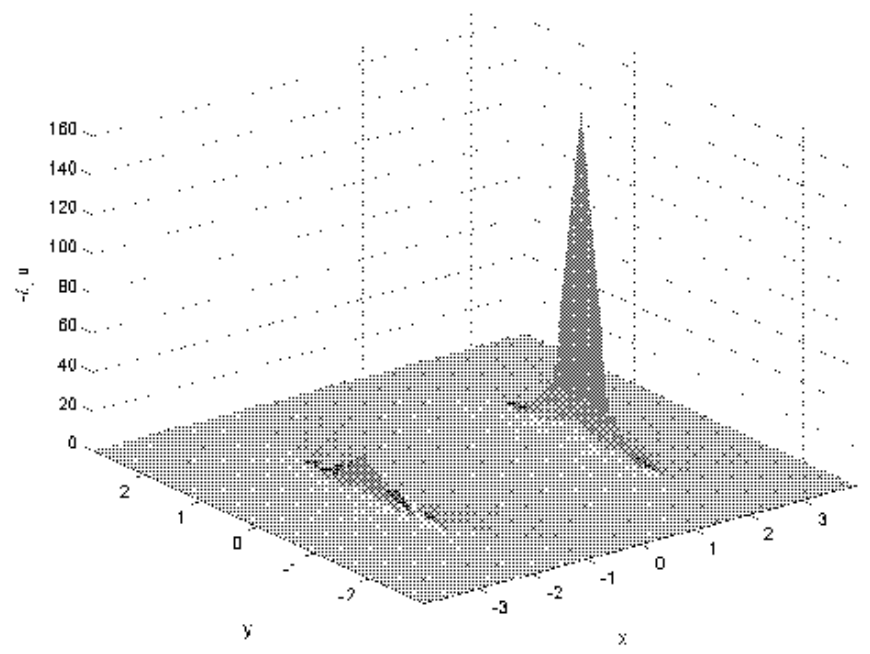

Figure 17. The $x$-derivative of the regularized dKP-I solution with initial data (5.1) and $\sigma=0.01$, plotted at the time $t=0.3$.

on the $x$-axis (note that we plot here $-\partial_{x} u$ ). As expected the maximum of the derivative is of the order $1 / \sigma$, and it is significantly bigger in the wave front for $x>0$, where the tails form. This can also be inferred from Fig. 18, where the dKP-I solution is plotted on the $x$-axis for several values of the time. We observe
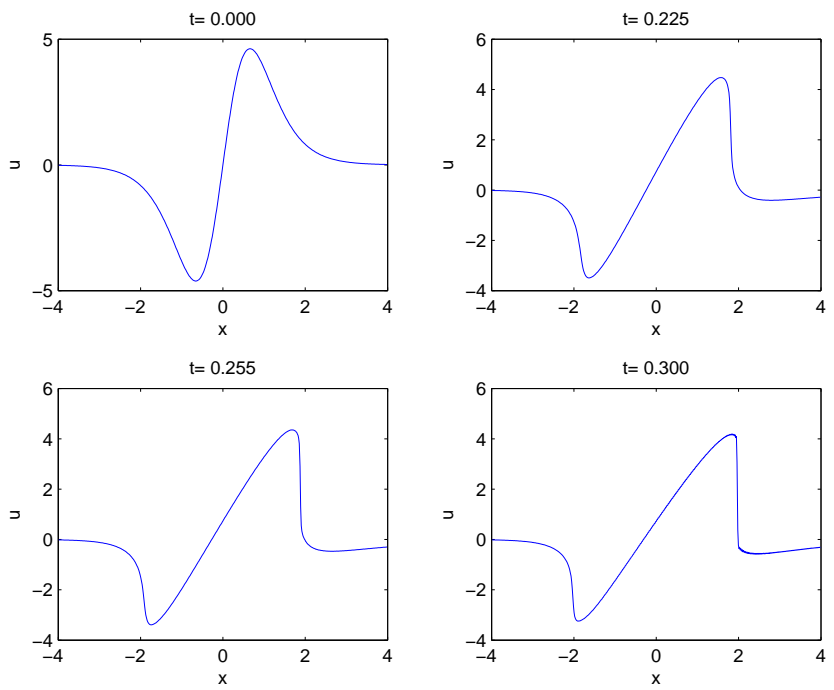

FiguRE 18. Solution to the regularized dKP-I equation with initial data (5.1) and $\sigma=0.01$, plotted on the $x$-axis for several values of $t$.

that the gradient catastrophe is reached for $x>0$ roughly at the time $t_{c} \approx 0.23$. For $t \geq t_{c}$, the gradient remains more or less unchanged due to the implemented 
dissipation in (5.7). The gradient catastrophe for the second wave front for $x<0$ is reached roughly for $t \approx 0.3$.

For $\lambda=1$, i.e. the KP-II case, the situation is similar, but the tails are now directed towards $x \rightarrow-\infty$. The gradient catastrophe is now reached first in the wave front for $x<0$. This is due to the fact that the dKP equation (1.8), as well as its dissipative regularization (5.7), are invariant under the simultaneous change of $x \rightarrow-x, u(t) \rightarrow-u(t)$, and $\lambda \rightarrow-\lambda$, for $t \geq 0$. For initial data of the type (5.1) which are odd in $x$, i.e. $u_{I}(-x, y)=-u_{I}(x, y)$, one consequently obtains the dKP-II solution $u_{+}(t, x, y)$ from the dKP-I solution $u_{-}(t, x, y)$, with the same initial data, by identifying $u_{+}(t, x, y)=-u_{-}(t,-x, y)$.

5.2. KP oscillations. In the following we shall study the oscillations for small, but still non-zero, $\epsilon \ll 1$ in the solution of (1.1).

In the closely related $\mathrm{KdV}$ case this limiting regime is rather well understood, see, e.g., 15, 29, 30, 39]: Roughly speaking, the corresponding analytical approach consists of the following steps: After the breaking in the corresponding Hopf solution (5.4), an oscillatory zone is identified via the solution of the so-called Whitham equations, which depend only on the slow coordinates $x$ and $t$. The corresponding approximate solution to the $\mathrm{KdV}$ equation in this region is consequently given in terms of theta functions, evaluated on the fast coordinates $X=x / \epsilon, T=t / \epsilon$, whereas the branch points of the underlying Riemann surface are determined via Whitham's modulation equations. Outside the oscillatory zone, the KdV solution is approximated by the corresponding solution of the Hopf equation. For a comparison of this asymptotic solution with a numerical KdV solution see [16].

There are several obstacles to overcome when one tries an analogous approach in the KP case. First, one should keep in mind that the formal dispersionless KdV model, i.e. the Burger's equation (5.2), is a first order PDE which can be integrated by the method of characteristics. On the other hand, the corresponding dKP equation, is a second order PDE. Although the dKP equation is completely integrable and thus explicit solutions are known, there exists no general procedure of integrating this equation for generic initial data so far. Secondly, the Whitham equations in the $\mathrm{KdV}$ case can be brought, via the hodograph transform, into the so-called Riemann linear form. They can then be again solved (at least implicitly) for generic initial data. Thus all quantities entering the theta functional solution, as for instance the phase, are known explicitly via their dependence on the branch points of the Riemann surface defined by the solution of the Whitham equations.

Remark 5.3. In the KP case, the corresponding Whitham equations were solved formally in 28]. So far, however, it is not clear how to bring this solution into a form which is convenient for a numerical simulation. This is especially true for the non-monotonous initial data with two inflection points we are considering here, a case which has not even been studied in the much better understood KdV setting. (Note that the two inflection points are needed to satisfy the constraint (2.10).) In addition it would be necessary to have an explicit formula for the corresponding phase entering the theta function to be able to compare the asymptotic solution to the exact solution in the Whitham zone.

We hope that the numerical results presented in this paper help to develop a similar asymptotic description as in the KdV case which will be the subject of further research. 
Now, we shall first show (numerically) that the solution of the dKP equation gives the correct limiting behavior as $\epsilon \rightarrow 0$ before breakup. To this end we compare the dKP-I solution $u_{\mathrm{dKP}}(t)$, with initial data (5.1), and the corresponding KP-I solution $u_{\mathrm{KP}}(t)$ at times $t \leq 0.2$, i.e. up to times close to (but still before) the appearance of the first shock in the dKP-I solution. We shall consider values of $\epsilon$ between 0.1 and 0.01 , with $N_{x}=2^{11}, N_{y}=2^{7}$, and $\Delta t=2 \times 10^{-5}$. Note that we solve here the dKP equation without dissipative regularization since we stop the computation before the appearance of the gradient catastrophe. We also remark that this is possible with a relative mass conservation of $10^{-4.39}$.

We find that the difference between the solutions $u_{\mathrm{dKP}}(t), t<t_{c}$, and $u_{\mathrm{KP}}(t)$ increases monotonically with time. In the considered range of $\epsilon$ and for $t=0.2$, the $L^{2}$ norm difference $\Delta_{2}$, defined in (4.16), decreases roughly like $\mathcal{O}\left(\epsilon^{3 / 2}\right)$, as $\epsilon \rightarrow 0$. More precisely we find that the data can be fitted by a straight line $-\log _{10} \Delta_{2}=-a \log _{10} \epsilon+b$, with $a=1.45$ and $b=1.30$, as can be seen in Fig. 19. The correlation coefficient is found to be $r=0.999$, the standard error for $a$ is $\sigma_{a}=0.056$.

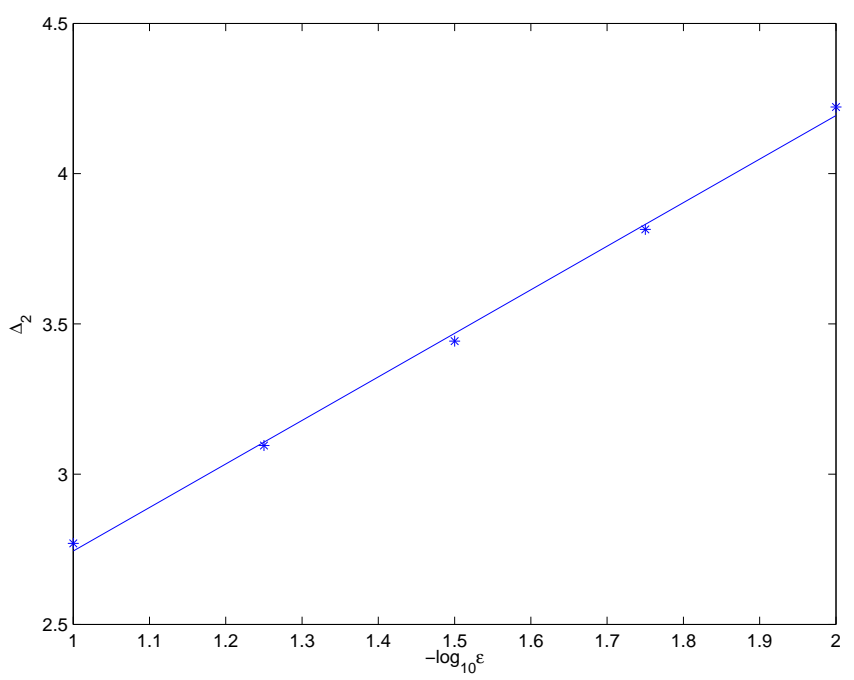

Figure 19. Error $\Delta_{2}$ for several values of $\epsilon$. The data can be fitted by least square analysis with a straight line $-\log _{10} \Delta_{2}=$ $-a \log _{10} \epsilon+b$ with $a=1.45$ and $b=1.30$. The correlation coefficient is $r=0.999$, the standard error for $a$ is $\sigma_{a}=0.056$.

Remark 5.4. Note that the $\mathcal{O}\left(\epsilon^{3 / 2}\right)$ error in $\Delta_{2}$ fits with our results of Section 4 There we numerically found that the $\Delta_{2}$ error for asymptotically small solutions, i.e. solutions of the order $\mathcal{O}(\epsilon)$, decays like $\mathcal{O}\left(\epsilon^{5 / 2}\right)$ for small $\epsilon \ll 1$.

For the corresponding $L^{\infty}$ norm difference $\Delta_{\infty}$ we find numerically that the error roughly decreases like $\epsilon$. More precisely again, we get (see Fig. 20), that the data can be fitted by a straight line $-\log _{10} \Delta_{2}=-a \log _{10} \epsilon+b$ with $a=.96$ and $b=-0.31$. The correlation coefficient is found to be $r=0.993$, the standard error for $a$ is $\sigma_{a}=0.099$. Notice that at $t=0.2$, the first oscillations of the KP solutions appear as can be seen below. This leads to considerably large values of $\Delta_{\infty}$ since the solution to the dKP equation will never show oscillations. 


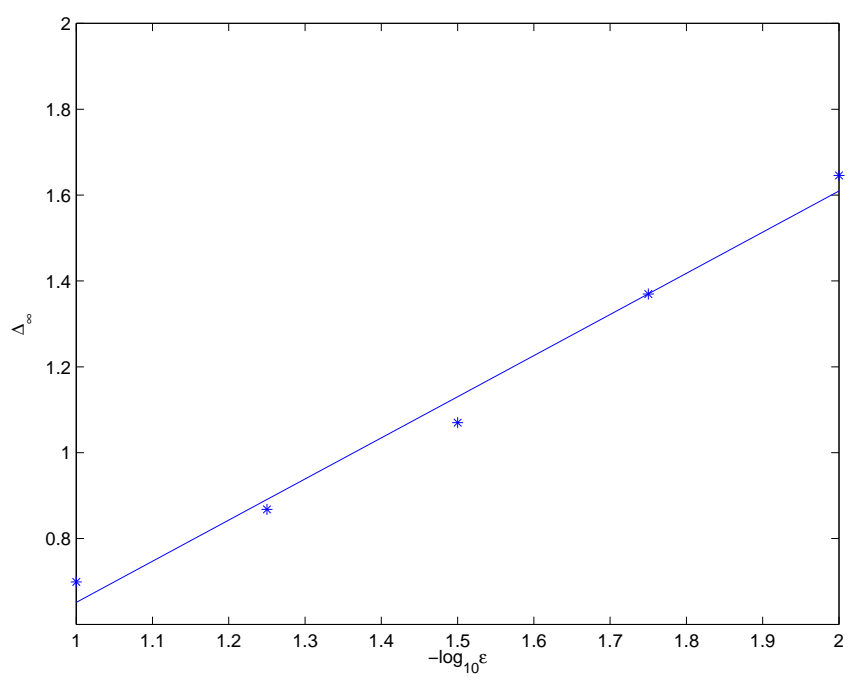

Figure 20. Error $\Delta_{\infty}$ for several values of $\epsilon$. The data can be fitted by least square analysis with a straight line $-\log _{10} \Delta_{\infty}=$ $-a \log _{10} \epsilon+b$ with $a=0.96$ and $b=-0.31$. The correlation coefficient is $r=0.993$, the standard error for $a$ is $\sigma_{a}=0.099$.

For times $t \geq t_{c}$, i.e. beyond the onset-time of the first gradient catastrophe, we shall study the KP-I solution, corresponding to the initial condition (5.1), for $0.01 \leq \epsilon \leq 0.1$ and times $t \leq 0.4$. The computations are carried out with $N_{x}=2048$ to $N_{x}=8192, N_{y}=128, L_{x}=L_{y}=5$, and $\Delta t=2 \times 10^{-5}$ to $\Delta t=4 \times 10^{-6}$. Thereby we observe a relative mass conservation of the order of $10^{-4}$ to $10^{-3}$. The following observations are now in order:

- It can be seen in Fig. 21] for the KP-I case that oscillations mainly form in the region of large (spatial) gradients appearing in the dKP solution given above.

- As in the KdV case the number of oscillations increases with decreasing $\epsilon$, whereas the wavelength decreases, see Fig. 22

- As expected there are two oscillatory regions, one for $x<0$ and one for $x>0$, both of which are shown in detail in Fig. 223 To get a complementary view of the rapid oscillations in this region a contour plot of the same situation is shown in Fig. 24

- The oscillations are always at most rapid on the $x$-axis, see Fig. 25] and the results of the next subsection.

We note that the fast oscillations are numerically well resolved, as can be inferred from Fig. 26] which shows two enlarged sections of the oscillatory zone. Moreover we observe that the time evolution of the initial data (5.1) is as expected from the analysis of the regularized dKP equation above.

To obtain more insight, we shall focus in Fig. 27 on the KP-I solution plotted at $y=0$, i.e. where the rapid oscillations appear first and where they are the most pronounced. We note that the first oscillation appears at the time $t \approx 0.22$, for positive values of $x \in \mathbb{R}$, i.e. shortly before reaching the gradient catastrophe of the dKP equation. On the other hand at time $t \approx 0.3$, the first oscillation appears 


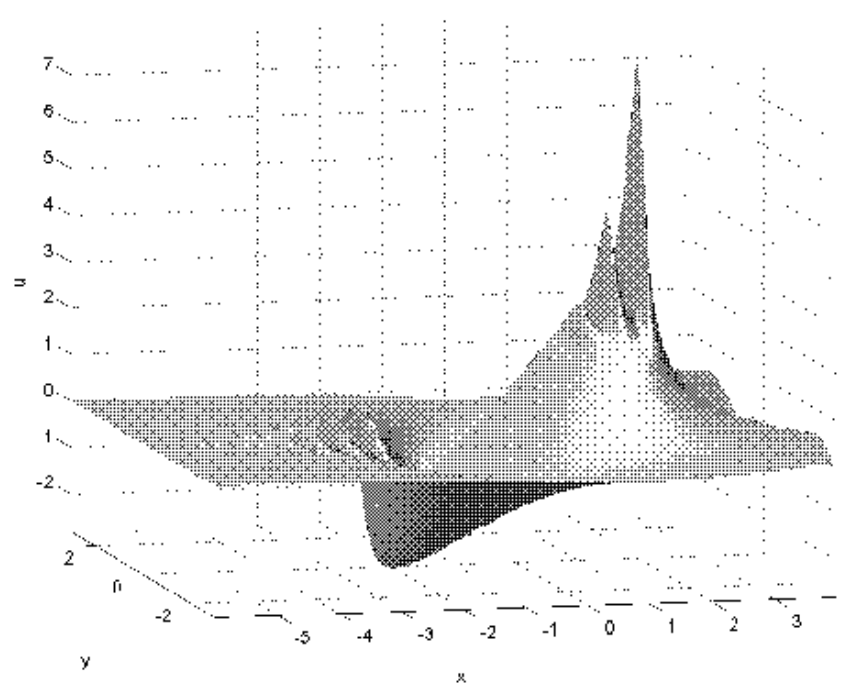

Figure 21. Solution of the KP-I equation obtained from the initial data (5.1) for $\epsilon=0.1$, at time $t=0.4$.

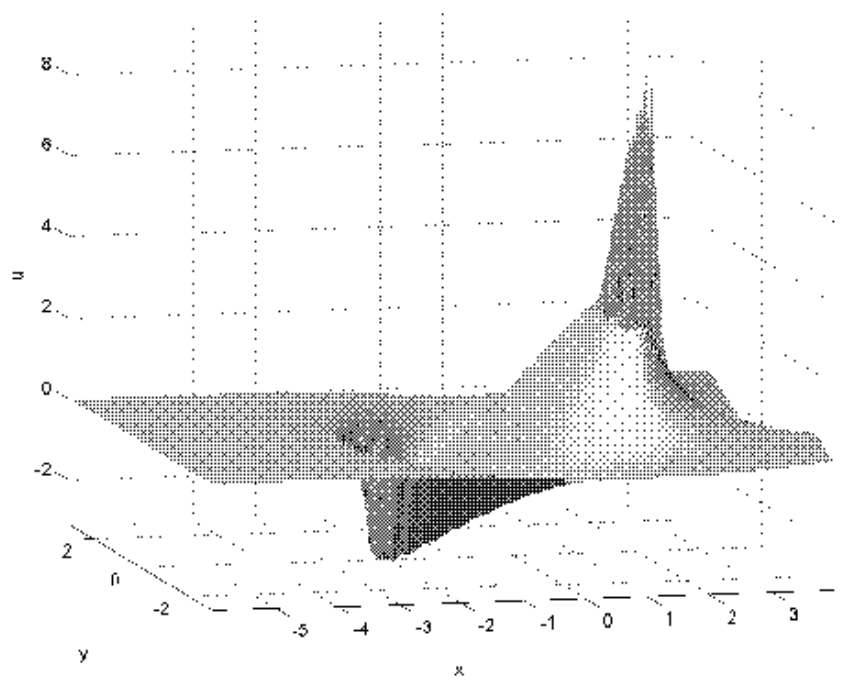

Figure 22. Solution of the KP-I equation obtained from the initial data (5.1) for $\epsilon=0.01$, at time $t=0.4$.

at a negative value of the $x$-axis. Again this is shortly before a shock in the second wave front is reached. Both regions then develop more and more oscillations as time goes on until finally the situation described above and shown in Fig. 25] and Fig. 26] is reached. In Fig. 23] and Fig. 24] one can also see the $y$-dependence of the observed oscillations. One recognizes that although they are mainly concentrated on the $x$-axis, there are some oscillations for small $0<|y| \ll 1$ (see also the results of the next subsection). 

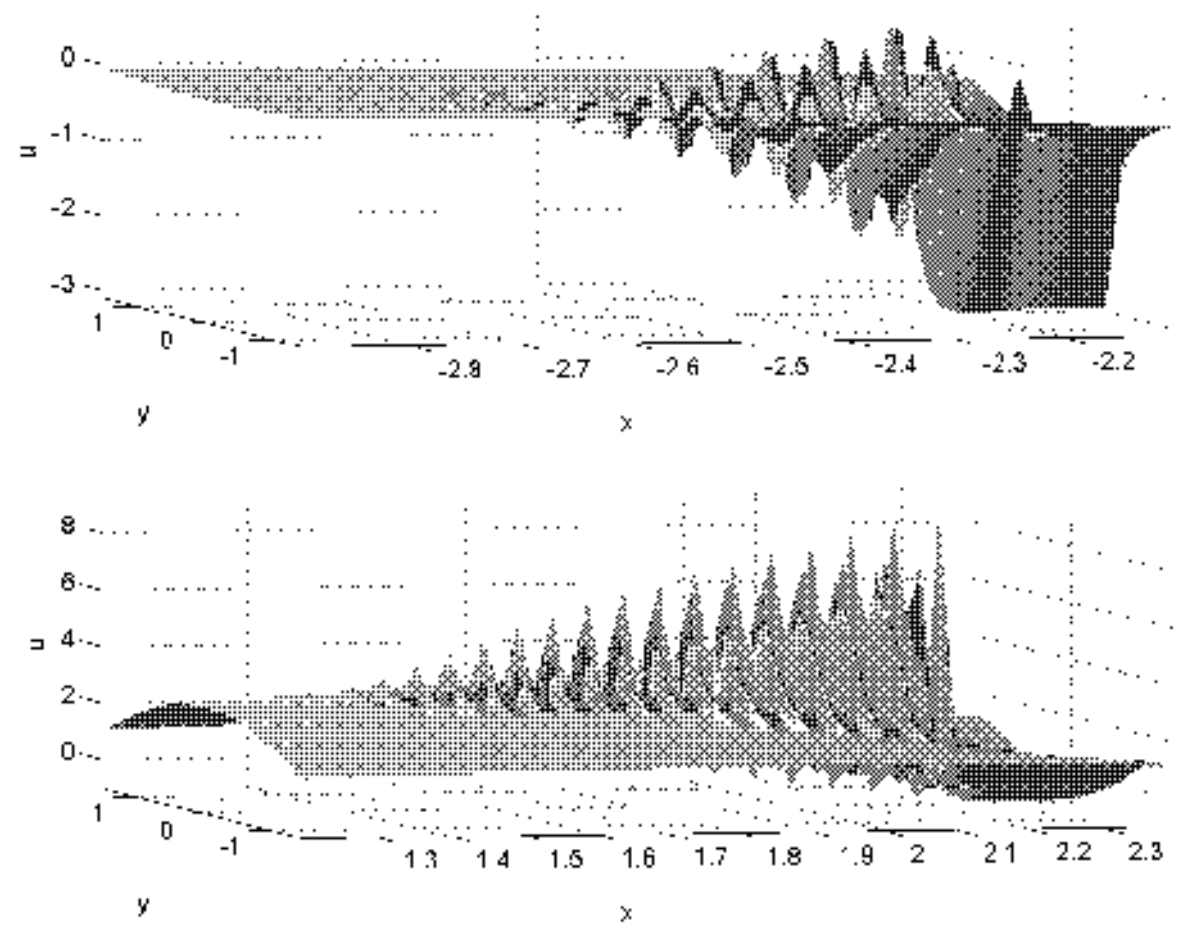

Figure 23. Solution of the KP-I equation obtained from the initial data (5.1) for $\epsilon=0.01$, at time $t=0.4$.

Remark 5.5. In the corresponding situation for the KdV equation, the oscillatory zone shrinks with $\epsilon$. The same is the case for the KP model as can be inferred from Fig. 28 and Fig. 29] Note that this shrinking is also present in the $y$-direction.

In contrast to the dKP equation, the KP equation is not invariant under the transformation $x \rightarrow-x, u(t) \rightarrow-u(t)$, and $\lambda \rightarrow-\lambda$. Thus the rapid oscillations for small $\epsilon \ll 1$ in the KP-II equation will be rather different from the KP-I case, as can be seen from Fig. 30. Here, the tails directed to $x \rightarrow-\infty$ are clearly visible as well as the strong oscillations for $x<0$. On the other hand there are virtually no oscillations for $x>0$. For the sake of comparison we plot in Fig. 31] the situation for both KP models, i.e. for the cases $\lambda= \pm 1$, at $y=0$ and $t=0.4$. Whereas there are rather strong oscillations near both breaking wave fronts in the KP-I case $(\lambda=-1)$, the KP-II case $(\lambda=1)$ enhances oscillations for $x<0$ while for for $x>0$ they are more or less suppressed.

5.3. Dependence on the $y$-coordinate. As already mentioned, the KP equation was originally derived to describe quasi one-dimensional (dispersive) wave phenomena with only weak transverse effects. Thus physically interesting solutions will have a rather weak dependence on the coordinate $y \in \mathbb{R}$. Mathematically it is nevertheless interesting to study the effects of a stronger $y$-dependence. In this subsection we will thus have a closer look on this dependence in the context of low-dispersion. 

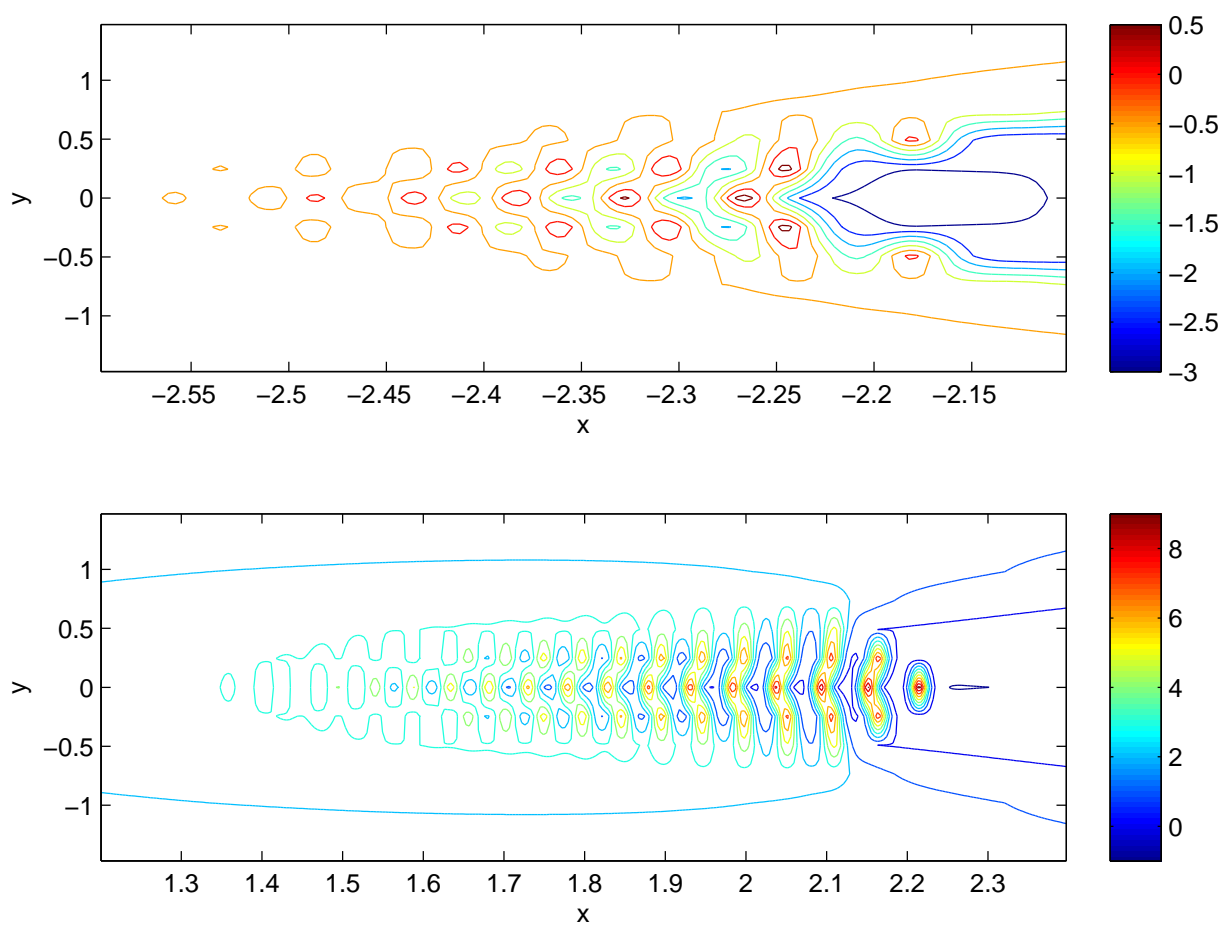

Figure 24. Solution of the KP-I equation obtained from the initial data (5.1) for $\epsilon=0.01$, at time $t=0.4$

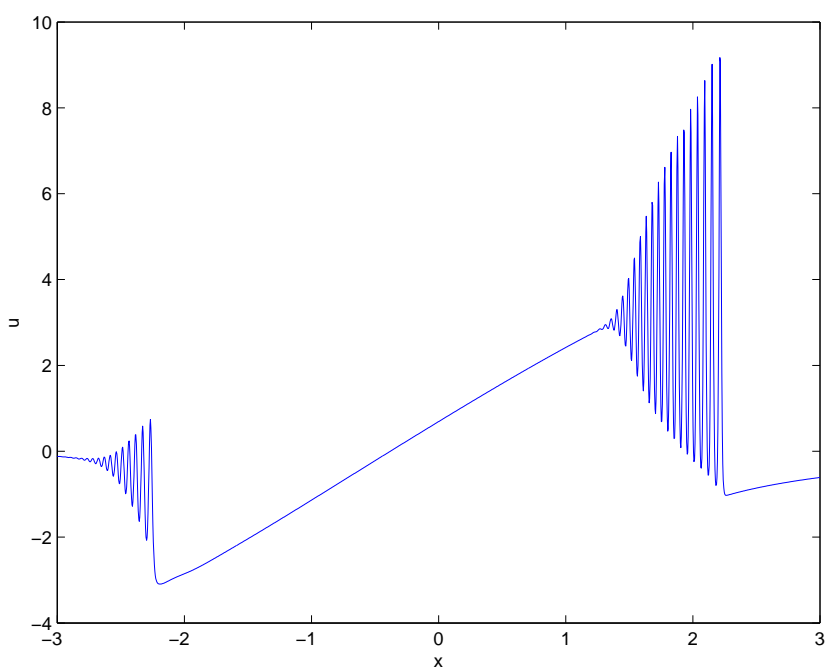

Figure 25. Solution of the KP-I equation obtained from the initial data (5.1) for $\epsilon=0.01$, at time $t=0.4$ and $y=0$. 

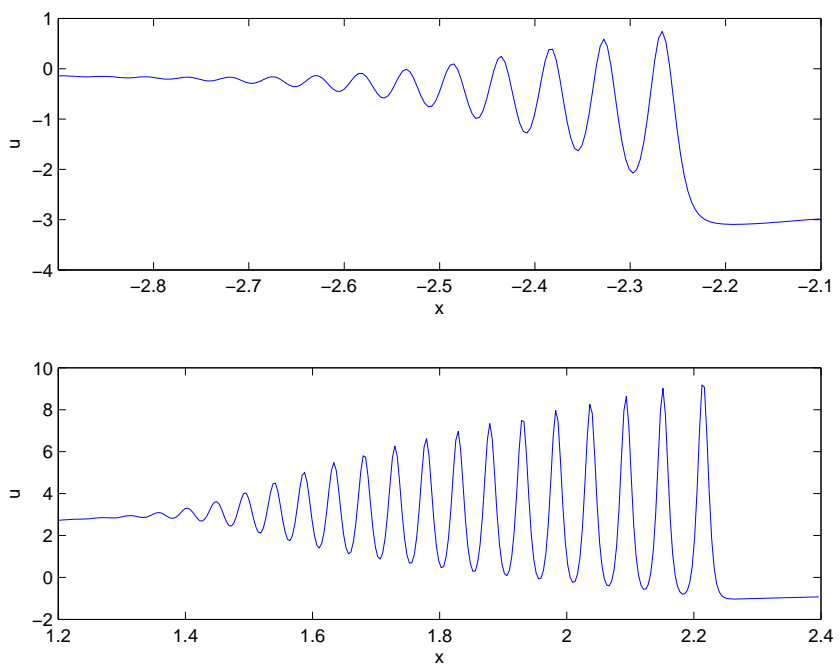

Figure 26. Solution to the KP-I equation obtained from the initial data (5.1) for $\epsilon=0.01$, at time $t=0.4$ and $y=0$.
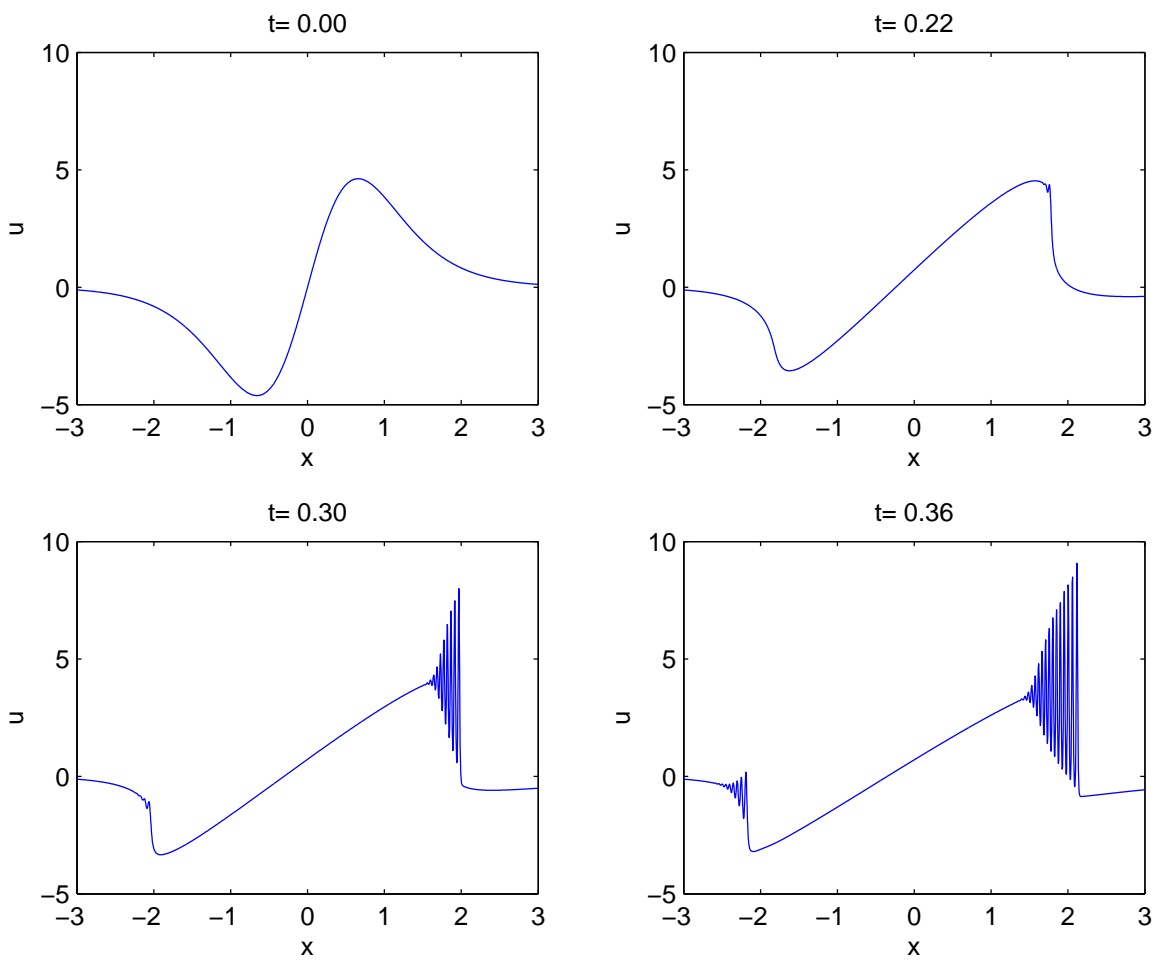

Figure 27. Solution of the KP-I equation obtained from the initial data (5.1) for $\epsilon=0.01$ for several values of the time. 


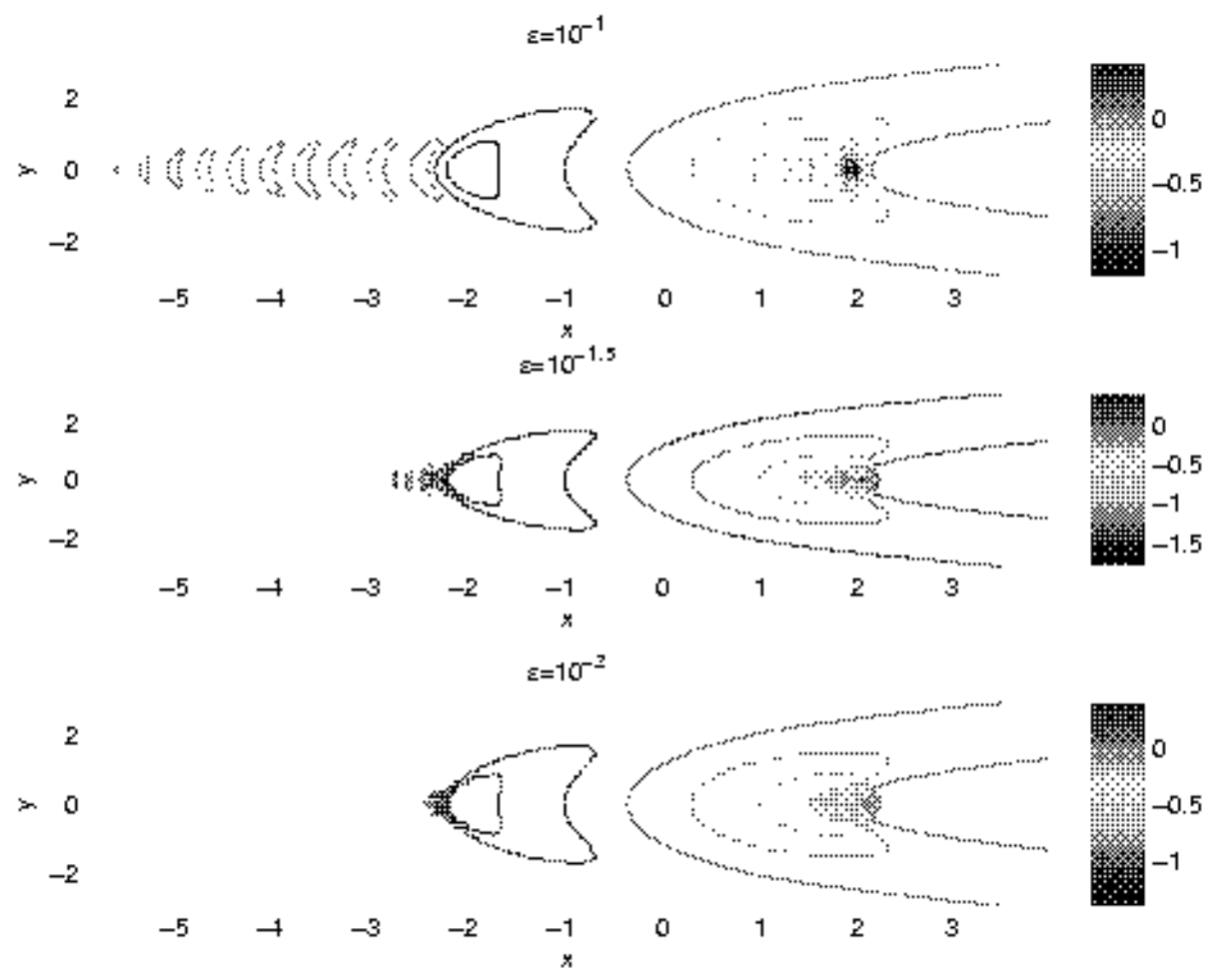

Figure 28. Solution of the KP-I equation obtained from the initial data (5.1) at time $t=0.4$ for several values of $\epsilon$.

Recalling the analysis of the Green's function for the linear part of the KP equation, given in Section 2.1, it appears that only the Airy-part leads to oscillations, whereas the term $\propto \partial_{y y} u$ is responsible for the formation of tails. On the other hand, the gradient catastrophe in the dKP model is the result of the nonlinear term $\propto u \partial_{x} u$, as in the case of the Hopf equation. Consequently one expects for initial data with a non-trivial $y$-dependence that oscillations can only appear in a small vicinity of the $x$-axis, i.e. before the formation of tails takes over. This behavior has already been observed in the subsection above and it is further supported by Fig. 32 which shows the KP solution for $\epsilon=0.01$ and $t=0.4$ in the vicinity of the $x$-axis. To study the effect of the dependence on the $y$-coordinate in more detail, we consider again initial data of the form (5.1). However we shall now evaluate them at $R=R_{\nu}$, given by

$$
R_{\nu}^{2}=x^{2}+\nu y^{2}
$$

where now $\nu \in \mathbb{R}_{0}^{+}$is a deformation parameter. Obviously, for $\nu=0$, there is no $y$-dependence, and the KP model reduces to the KdV equation. The parameter $\nu$ thus allows for a continuous deformation of the $\mathrm{KdV}$ sector.

In the following we only consider the case $\epsilon=0.1$. The corresponding solution to the $\mathrm{KdV}$ equation can be seen in Fig. 33 For simplicity, we shall only compare solutions for different values of $\nu$ plotted at $y=0$, i.e. the region where the oscillations are the most pronounced. In Fig. 34 the solution to the KP-I equation with initial data (5.1), (5.10), is shown for several values of $\nu$. It can be seen that the case $\nu=0.01$ 

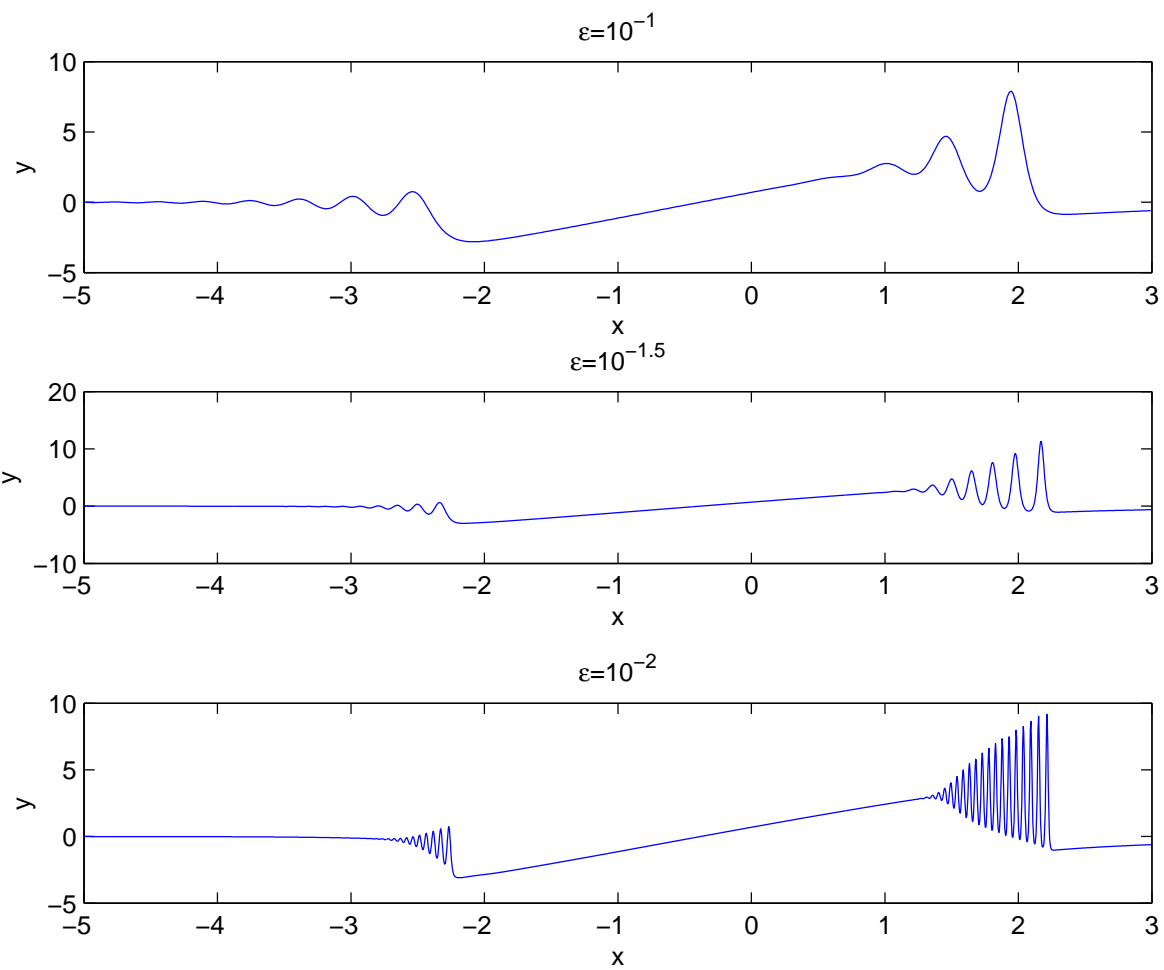

Figure 29. Solution of the KP-I equation obtained from the initial data (5.1) for several values of $\epsilon$, at time $t=0.4$ and $y=0$.

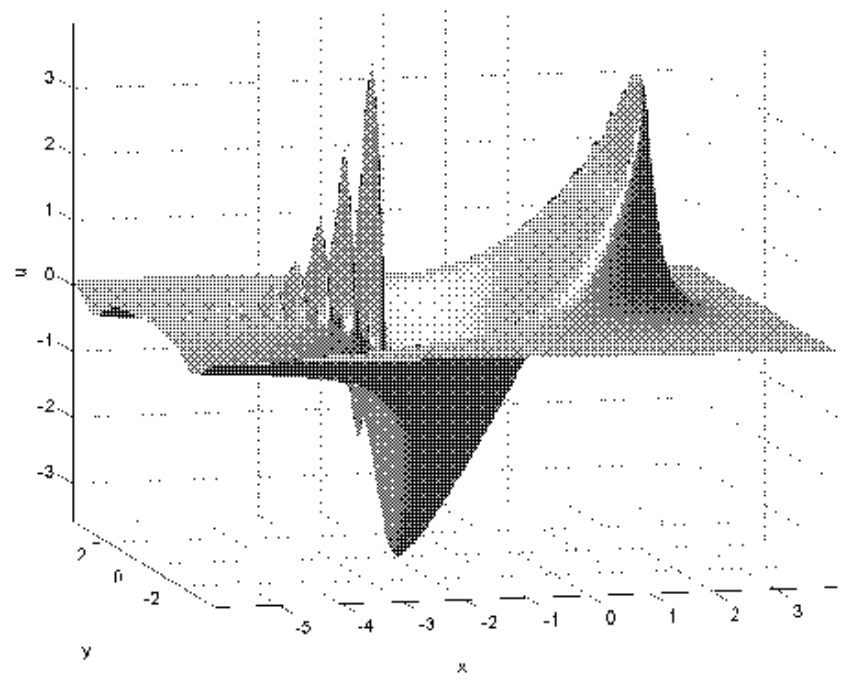

Figure 30. Solution of the KP-II equation obtained from the initial data (5.1) for $\epsilon=0.1$ at time $t=0.4$. 

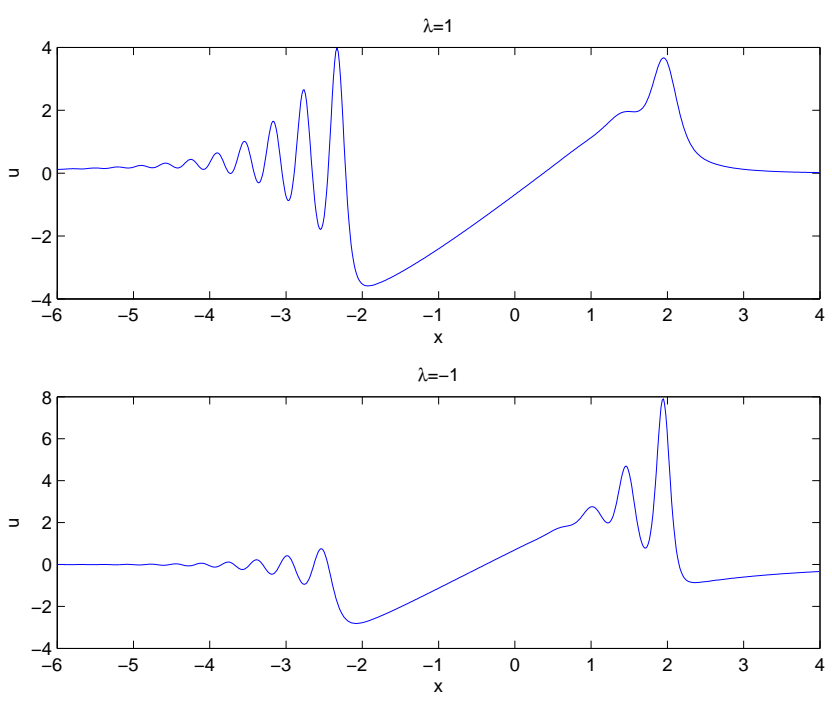

Figure 31 . Solutions to both KP models obtained from the same initial data (5.1), shown at time $t=0.4$ and $y=0$.
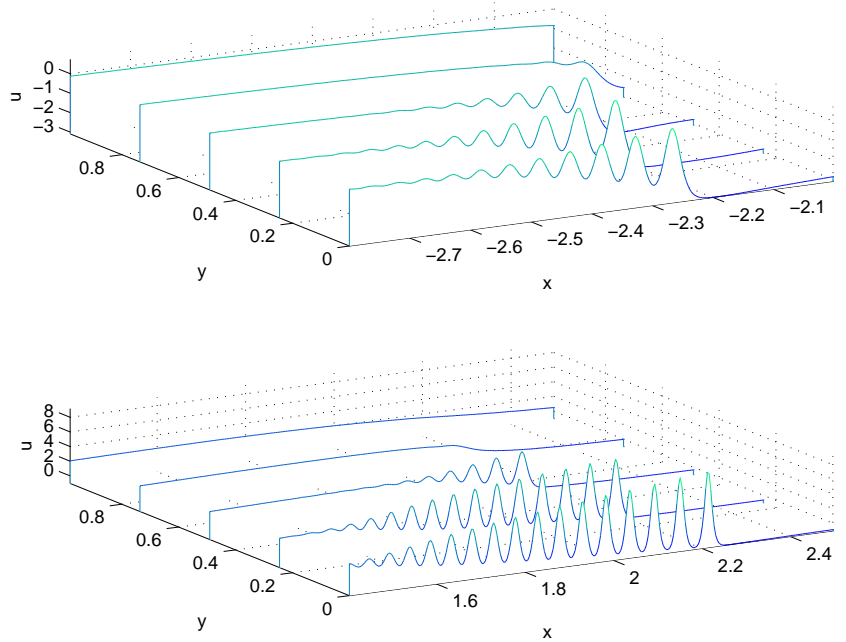

Figure 32. Solution of the KP-I equation obtained from the initial data (5.1) for $\epsilon=0.01$ at time $t=0.4$.

is very close to the $\mathrm{KdV}$ situation. Thus a continuous deformation of the $\mathrm{KdV}$ sector to a nontrivial $y$-dependence is possible, also numerically. Increasing $\nu$ has two effects: First we observe that the oscillations for $x<0$ are suppressed whereas the oscillations for $x>0$ are enhanced. This qualitative behavior has already been encountered above by comparing solutions of the KP-I and KP-II equation for the same initial data. Secondly, since a stronger $y$-dependence enhances the the formation of tails we observe that, the bigger $\nu$ is, the more mass is transferred 


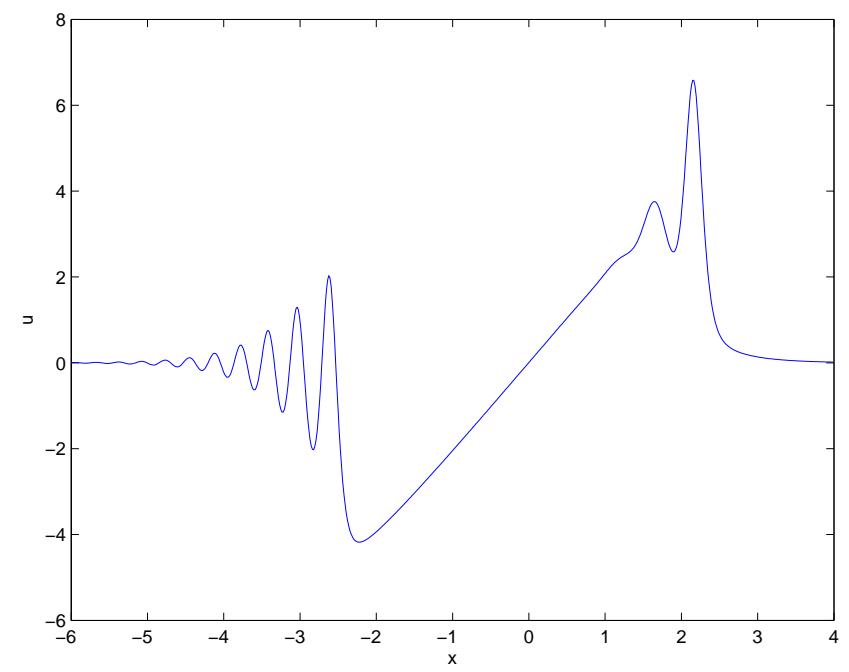

Figure 33. Solution of the KdV equation with $\epsilon=0.1$ obtained from the initial data (5.1) with (5.10) and $\nu=0$ for the time $t=0.4$.

from the initial wave pulse to these tails. This implies that less mass is available to form shocks in the dispersionless case and thus rapid oscillations for small $\epsilon$ are severely damped for large $\nu$, as is clearly visible in the case $\nu=10$.

Remark 5.6. In the case $\nu=10$ the periodic boundary conditions produces very strong echoes, as it is clearly visible in Fig. 35] This however is the only example considered here, where the echoes dominate the oscillations which we want to study.

Finally the corresponding transition in $\nu$ for the KP-II case $(\lambda=1)$ starting from a situation close to KdV is demonstrated in Fig. 36] There the oscillations for negative $x$ are enhanced whereas the oscillations for positive $x$ are suppressed as $\nu$ increases. For large values of $\nu$ we again find that more and more mass is transferred to the tails, i.e. pushed to $x \rightarrow-\infty$. In summary we find that the tails tend to suppress strong gradients, and thus shocks in the dKP equation, as well as the corresponding rapid oscillations in (1.1) for small $\epsilon$.

\section{REFERENCES}

1. M. J. Ablowitz and P. A. Clarkson, Solitons, nonlinear evolution equations and inverse scattering, Cambridge Univ. Press, Cambridge (1991).

2. M. J. Abramowiz and I. A. Stegun (Eds.), Handbook of Mathematical Functions with Formulas, Graphs, and Mathematical Tables, New York Dover 1972.

3. J. C. Alexander, R. L. Pego, and R. L. Sachs, On the transverse instability of solitary waves in the Kadomtsev-Petviashvili equation, Phys. Lett. A 226 (1997), 187-192.

4. C. Besse, N. Mauser, and H. P. Stimming, Numerical study of the Davey-Stewartson system, Math. Model. Numer. Anal. 38 (2004), no. 6, 1035-1054.

5. V. N. Bogaevskiū, On Korteweg-de Vries, Kadomtsev-Petviashvili, and Boussinesq equations in the theory of modulations, Zh. Vychisl. Mat. i Mat. Fiz. 30 (1990), no. 10, 14871501; translation in U.S.S.R. Comput. Math. and Math. Phys. 30 (1991), no. 5, 148-159.

6. M. Boiti, F. Pempinelli, and A. Pogrebkov, Properties of solutions of the KadomtsevPetviashvili I equation, J. Math. Phys. 35 (1994), issue 9, 4683-4718. 

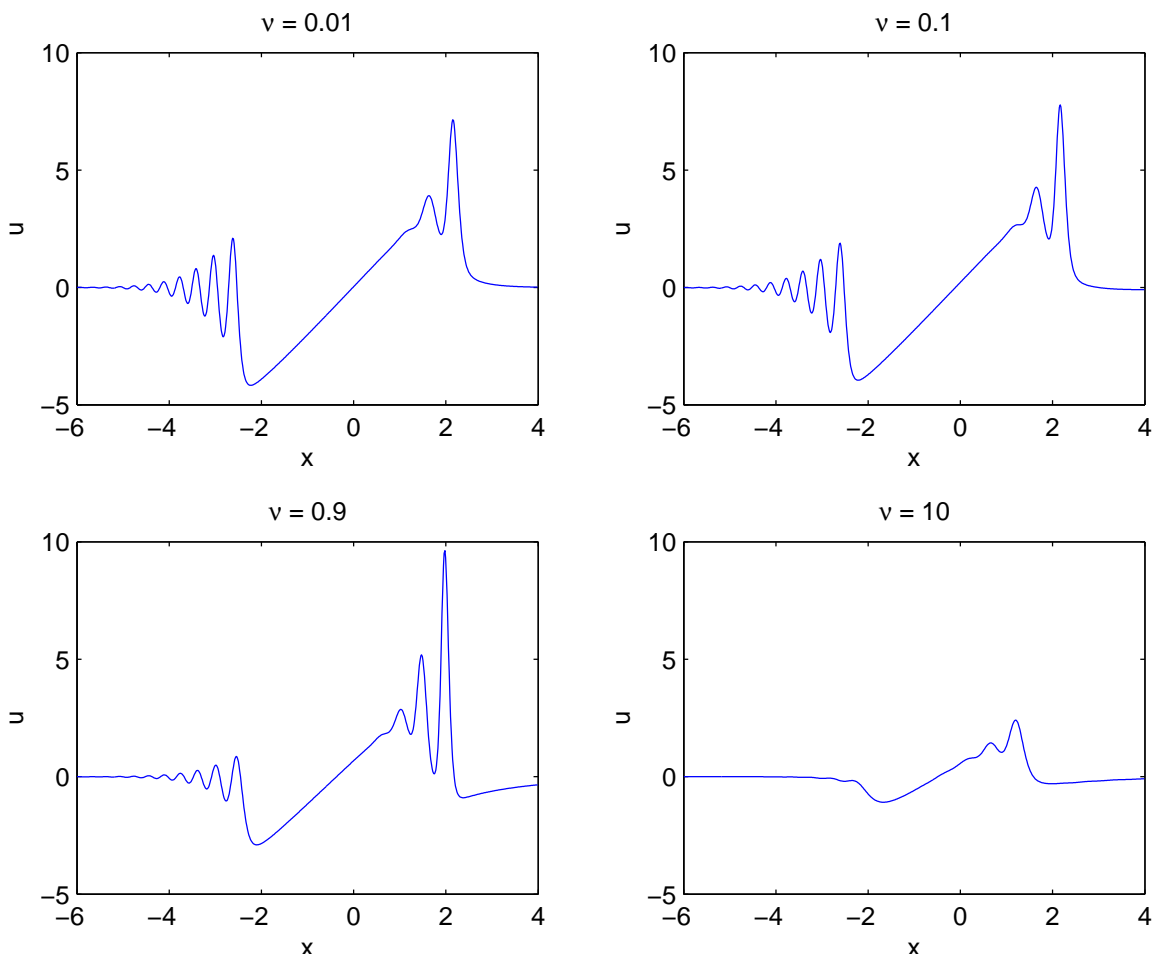

Figure 34. Solution of the KP-I equation obtained from the initial data (5.1), (5.10) for several values of $\nu$ at time $t=0.4$.

7. A. de Bouard and Y. Martel, Nonexistence of $L^{2}$-compact solutions of the KadomtsevPetviashvili II equation, Math. Annalen. 328 (2004), 525-544.

8. J. Bourgain, On the Cauchy problem for the Kadomtsev-Petviashvili equation, Geom. Funct. Anal. 3 (1993), no.4, 315-341.

9. C. Canuto, M. Y. Hussaini, and A. Quarteroni, and T. A. Zang, Spectral Methods in Fluid Dynamics, Springer-Verlag, Berlin 1988.

10. J. Colliander, C. Kenig, and G. Staffilani, Low regularity solutions for the KadomtsevPetviashvili I equation, Geom. Funct. Anal. 13 (2003), no.4, 737-794.

11. T. Driscoll, A Composite RungeKutta Method for the Spectral Solution of Semilinear PDEs, J. Comput. Phys. 182 (2002), issue 2, 357-367.

12. B. F. Feng, T. Kawahara, and T. A. Mitsui, conservative spectral method for several twodimensional nonlinear wave equations, J. Comput. Phys. 153 (1999), no. 2, 467-487.

13. E. V. Ferapontov and K. R. Khusnutdinova, Double waves in multi-dimensional systems of hydrodynamic type: the necessary condition for integrability, preprint, arXiv: nlin.SI/0412064

14. A. S. Fokas and L. Y. Sung, On the solvability of the $N$-wave, Davey-Stewartson and Kadomtsev-Petviashvili equations, Inverse Problems 8 (1992), 673-708.

15. T. Grava and Fei-Ran Tian, The generation, propagation, and extinction of multiphases in the KdV zero-dispersion limit, Comm. Pure Appl. Math. 55 (2002), no. 12, 1569-1639.

16. T. Grava and C. Klein, Numerical solution of the small dispersion limit of Korteweg de Vries and Whitham equations, preprint, arXiv: math-ph/0511011

17. R. H. Hardin and F. D. Tappert, Applications of the split-step Fourier method to the numerical solution of nonlinear and variable coefficient wave equations, SIAM review, Chronicle 15 (1973) 423.

18. T. Y. Hou, J. S. Lowengrub, and M. J. Shelley, Removing the Stiffness from Interfacial Flows with Surface Tension, J. Comp. Phys. 114 (1994) 312-338. 


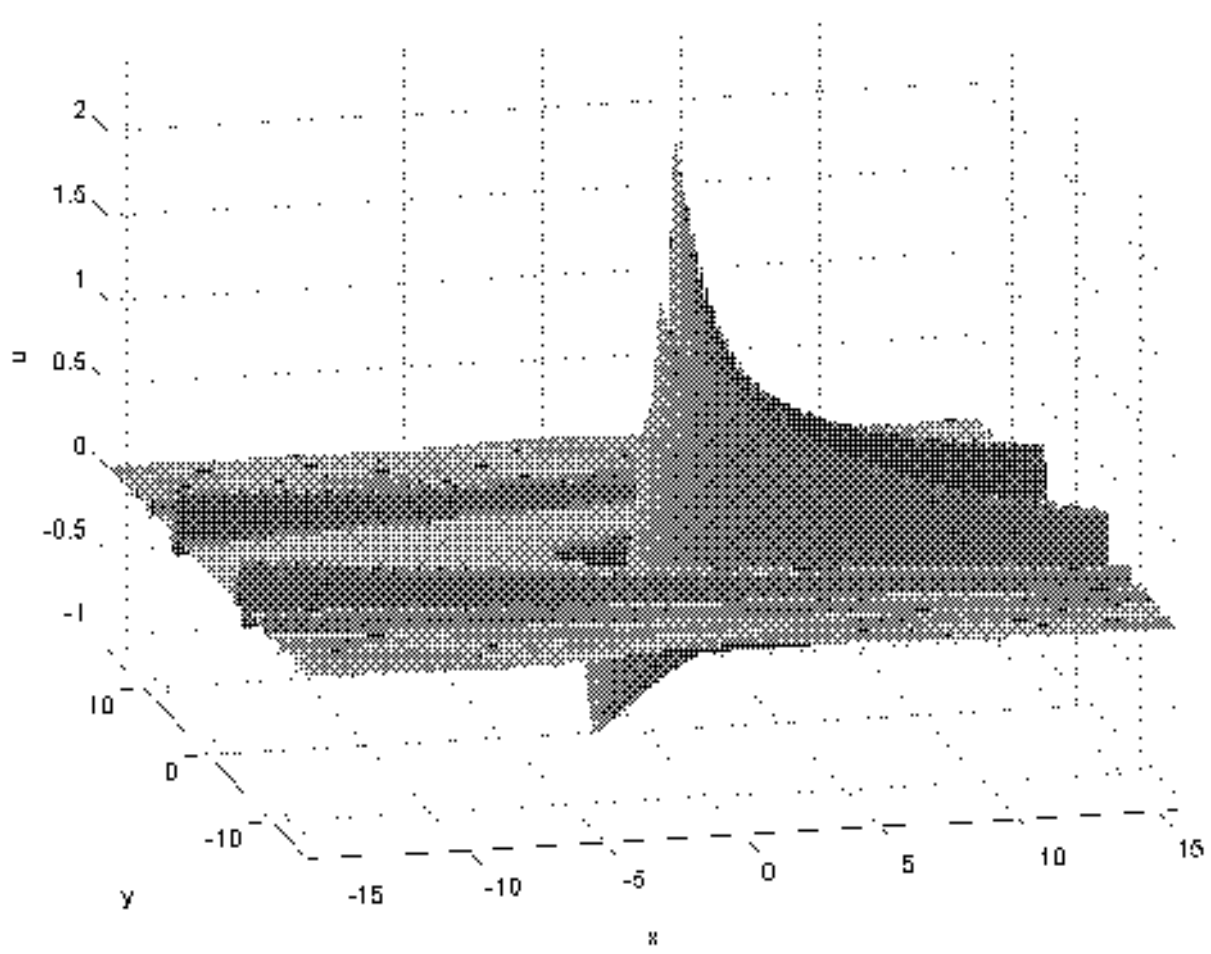

FiguRE 35. Solution of the KP-I equation obtained from the initial data (5.1) with (5.10) and $\nu=10$ at time $t=0.4$.

19. G. Huang, V. A. Makarov, and M. G. Velarde, Two-dimensional solitons in Bose-Einstein condensates with a disk-shaped trap, Phys. Rev. A 67 (2003), 23604-23616.

20. E. Infeld, A. Senatorski, and A. A. Skorupski, Numerical simulations of KadomtsevPetviashvili soliton interactions, Phys. Rev. E 51 (1995), 3183-3191.

21. E. Infeld, A. A. Skorupski, and G. Rowlands, Instabilities and oscillations of one- and twodimensional Kadomtsev-Petviashvili waves and solitons. II. Linear to nonlinear analysis. R. Soc. Lond. Proc. Ser. A (Math. Phys. Eng. Sci.) 458 (2002), no. 2021, 1231-1244.

22. R. S. Johnson, The classical problem of water waves: a reservoir of integrable and nearly integrable equations, J. Nonl. Math. Phys. 10 (2003) 72-92.

23. C. A. Jones and P. H. Roberts, Motions in a Bose condensate, IV: Axisymmetric solitary waves, J. Phys. A Math. Gen. 15 (1982), 2599-2619.

24. B. B. Kadomtsev and V. I. Petviashvili, On the stability of solitary waves in weakly dispersing media, Sov. Phys. Dokl. 15 (1970), 539-541.

25. D. Kaya and E. M. Salah, Numerical soliton-like solutions of the potential KadomtsevPetviashvili equation by the decomposition method, Phys. Lett. A 320 (2003), no. 2-3, 192-199.

26. Y. Kodama, A method for solving the dispersionless KP hierarchy and its exact solutions, Phys. Lett. A 129 (1988), no 4, 223-226.

27. Y. Kodama and J. Gibbons, A method for solving the dispersionless KP hierarchy and its exact solutions. II, Phys. Lett. A 135 (1989), no. 3, 167-170.

28. I. M. Krichever, The averaging method for two-dimensional integrable equations, Funktsional. Anal. i Prilozhen. 22 (1988), no. 3, 37-52; translation in Funct. Anal. Appl. 22 (1989), no. 3, 200-213.

29. P. D. Lax and C. D. Levermore, The small dispersion limit of the Korteweg de Vries equation I,II,III, Comm. Pure Appl. Math. 36 (1983), 253-290, 571-593, 809-830. 

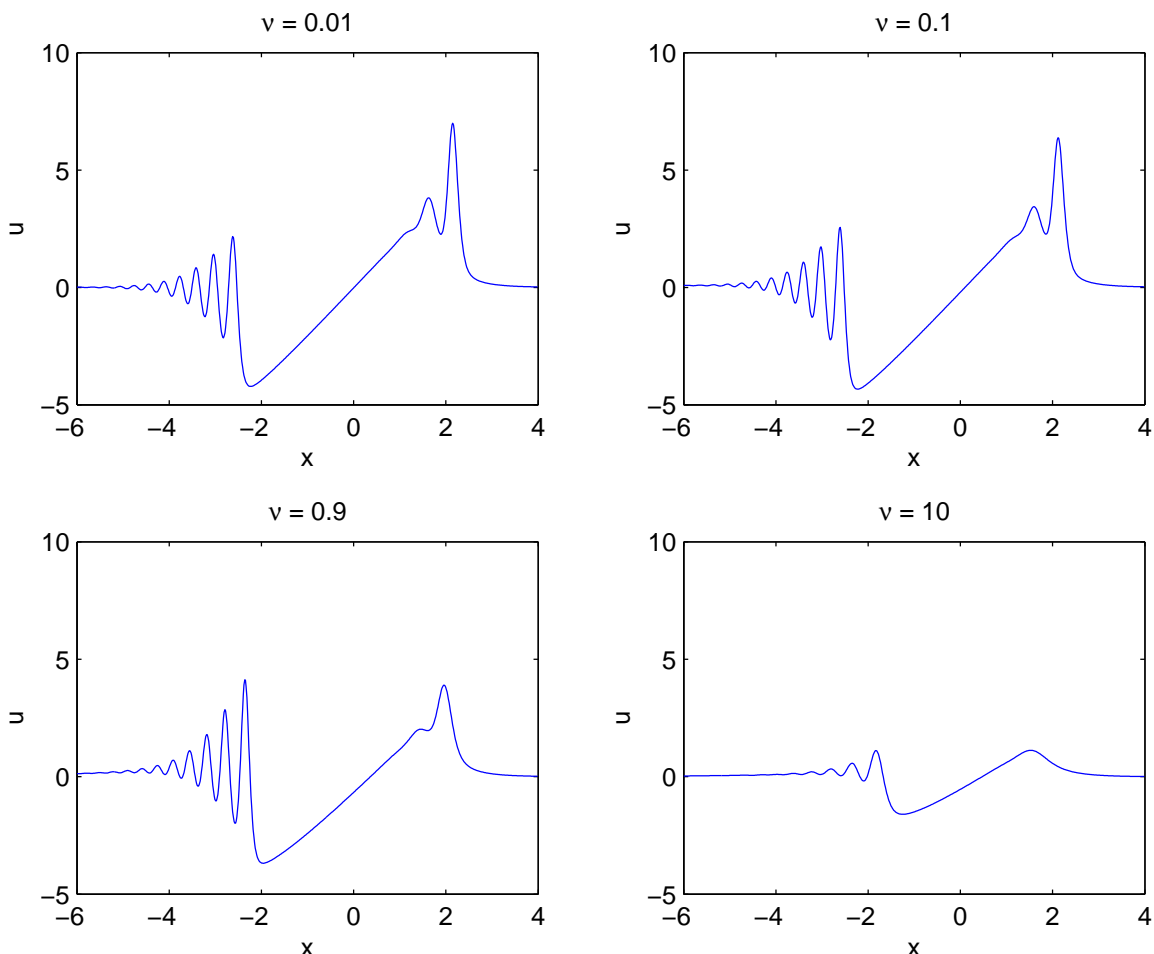

Figure 36. Solution of the KP-II equation obtained from the initial data (5.1) with (5.10) for several values of $\nu$ at time $t=0.4$.

30. C. D. Levermore, The hyperbolic nature of the zero dispersion KdV limit, Comm. Part. Diff. Equ. 13 (1988), no. 4, 495-514.

31. D. W. McLaughlin and J. A. Strain, Computing the weak limit of KdV, Comm. Pure Appl. Math. 47 (1994), no. 10, 1319-1364.

32. L. Molinet, J. C. Saut, and N. Tzvetkov, Well-posedness and ill-posedness results for the Kadomtsev-Petviashvili-I equation Duke Math. J. 115 (2002), no. 2, 353-384.

33. S. Novikov, S. V. Manakov, L. P. Pitaevskii, and V. E. Zakharov, Theory of Solitons: The Inverse Scattering Method Springer Monographs in Contemporary Mathematics, Springer 1984.

34. A. Senatorski and E. Infeld, Simulations of Two-Dimensional Kadomtsev-Petviashvili Soliton Dynamics in Three-Dimensional Space, Phys. Rev. Lett. 77 (1996), 2855-2858.

35. G. Schneider, Approximation of the Korteweg-de Vries equation by the nonlinear Schrdinger equation, J. Diff. Equ. 147 (1998), no. 2, 333-354.

36. C. Sulem and P. L. Sulem, The nonlinear Schrödinger equation, Applied Math. Sciences 139, Springer (1999).

37. H. Takaoka, Global well-posedness for the Kadomtsev-Petviashvili II equation, Discrete Contin. Dynam. Systems 6 (2000), 483-499.

38. H. Takaoka and N. Tzvetkov, On the local regularity of the Kadomtsev- Petviashvili-II equation, Internat. Math. Res. Notices 2001 (2001), no. 2, 77-114.

39. F. R. Tian, Oscillations of the zero dispersion limit of the Korteweg de Vries equations, Comm. Pure App. Math. 46 (1993), 1093-1129.

40. L. N. Trefethen, Spectral Methods in MATLAB, SIAM, Philadelphia 2000.

41. http://www.comlab.ox.ac.uk/oucl/work/nick.trefethen

42. S. Turitsyn and G. Falkovitch, Stability of magneto-elastic solitons and self-focusing of sound in antiferromagnets, Soviet Phys. JETP 62 (1985), 146-152. 
43. N. Tzvetkov, Bilinear estimates related to the KP equations, Journées Équations aux Dérivées Part. exp. no. XIX, Univ. Nantes, Nantes, 2000.

44. X. P. Wang, M. Ablowitz, and H. Segur, Wave collapse and instability of solitary waves of a generalized Kadomtsev-Petviashvili equation, Physica D 78 (1994), 241-265.

45. A. Wazwaz, A computational approach to soliton solutions of the Kadomtsev-Petviashvili equation, Appl. Math. Comput. 123 (2001), no. 2, 205-217.

46. V. E. Zakharov and A. E. Kuznetsov, Multi-scale expansion in the theory of systems integrable by the inverse scattering transform, Physica D 18 (1986), 455-463.

Max Planck Institute for Mathematics in the Sciences

E-mail address: klein@mis.mpg.de

Wolfgang Pauli Institute Vienna \& Faculty of Mathematics, Vienna University, NordBergstrasse 15, A-1090 Vienna, Austria

E-mail address: christof.sparber@univie.ac.at

Faculty of Mathematics, Vienna University, Nordbergstrasse 15, A-1090 Vienna, AusTRIA

E-mail address: peter.markowich@univie.ac.at 